\title{
Syntheses, characterization, density functional theory calculations, and activity of tridentate SNS zinc pincer complexes
}

\author{
John R. Miecznikowski \\ Fairfield University, jmiecznikowski@fairfield.edu \\ Follow this and additional works at: https://digitalcommons.fairfield.edu/chemistry-facultypubs \\ Eopyyerthe 2011 Elsevier
}


Chimica Acta. Changes resulting from the publishing process, such as peer review, editing, Briannectibns, strughlupal formatting, and other quality control mechanisms may not be reflected in

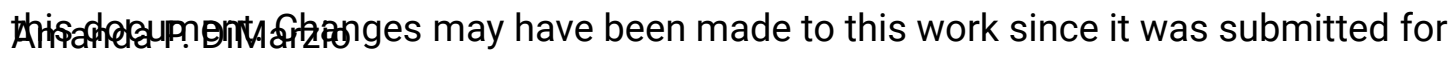
publication. A definitive version was subsequently published in Inorganica Chimica Acta, 2011, 376, 515-524. DOI:10.1016/j.ica.2011.07.021

See next page for additional authors

\section{Repository Citation}

Miecznikowski, John R.; Lo, Wayne; Lynn, Matthew A.; O'Loughlin, Brianne E.; DiMarzio, Amanda P.; Martinez, Anthony M.; Lampe, Lorraine; Foley, K. M.; Keilich, Lauren C.; Lisi, G. P.; Kwiecien, D. J.; Pires, C. M.; Kelly, W. J.; Kloczko, Nathan F.; and Morio, K. N., "Syntheses, characterization, density functional theory calculations, and activity of tridentate SNS zinc pincer complexes" (2011). Chemistry \& Biochemistry Faculty Publications. 12. https://digitalcommons.fairfield.edu/chemistry-facultypubs/12

\section{Published Citation}

Miecznikowski, J.R.; Lo, W.; Lynn, M.A.; O’Loughlin, B.E; DiMarzio, A.P; Martinez, A.M.; Lampe, L.; Foley, K.M.; Keilich, L.C.; Lisi, G.P.; Kwiecien, D.J.; Pires, C.M.; Kelly, W.J.; Kloczko, N.F.; Morio, K.N. "Syntheses, characterization, density functional theory calculations, and activity of tridentate SNS zinc pincer complexes." Inorganica Chimica Acta, 2011, 376, 515-524.

This item has been accepted for inclusion in DigitalCommons@Fairfield by an authorized administrator of DigitalCommons@Fairfield. It is brought to you by DigitalCommons@Fairfield with permission from the rightsholder(s) and is protected by copyright and/or related rights. You are free to use this item in any way that is permitted by the copyright and related rights legislation that applies to your use. For other uses, you need to obtain permission from the rights-holder(s) directly, unless additional rights are indicated by a Creative Commons license in the record and/or on the work itself. For more information, please contact digitalcommons@fairfield.edu. 


\section{Authors}

John R. Miecznikowski, Wayne Lo, Matthew A. Lynn, Brianne E. O'Loughlin, Amanda P. DiMarzio, Anthony M. Martinez, Lorraine Lampe, K. M. Foley, Lauren C. Keilich, G. P. Lisi, D. J. Kwiecien, C. M. Pires, W. J. Kelly, Nathan F. Kloczko, and K. N. Morio 
Syntheses, Characterization, Density Functional Theory Calculations, and Activity of Tridentate SNS Zinc Pincer Complexes

John R. Miecznikowski ${ }^{\mathrm{a} *}$; Wayne Lo ${ }^{\mathrm{b}}$; Matthew A. Lynn;

Brianne E. O'Loughlin ${ }^{\mathrm{a}}$; Amanda P. DiMarzio ${ }^{\mathrm{a}}$; Anthony M. Martinez;

Lorraine Lampe $^{\mathrm{a}}$; Kathleen M. Foley ${ }^{\mathrm{a}}$; Lauren C. Keilich ${ }^{\mathrm{a}}$; George P. Lisi ${ }^{\mathrm{a}}$; Daniel J.

Kwiecien ; Cristina M. Pires ; William J. Kelly ${ }^{\mathrm{a}}$, Nathan F. Kloczko ${ }^{\mathrm{a}}$, and Kaitlyn N. Morio ${ }^{\mathrm{a}}$

${ }^{a}$ Department of Chemistry and Biochemistry, Fairfield University, 1073 North Benson Road, Fairfield, CT 06824. U.S.A.

${ }^{\mathrm{b}}$ Department of Chemistry, Boston College, 140 Commonwealth Avenue, Chestnut Hill, MA 02467. U.S.A.

${ }^{c}$ Department of Science and Mathematics, National Technical Institute for the Deaf, Rochester Institute of Technology, 52 Lomb Memorial Drive, Rochester, NY 14623. U.S.A.

* Corresponding Author: Tel.: 1-(203) 254-4000 x 2125; Fax: 1-(203) 254-4034;

Email: jmiecznikowski@fairfield.edu 


\begin{abstract}
:
A series of tridentate SNS ligand precursors were metallated with $\mathrm{ZnCl}_{2}$ to give new tridentate SNS pincer zinc complexes. The zinc complexes serve as models for the zinc active site in Liver Alcohol Dehydrogenase (LADH) and were characterized with single crystal X-ray diffraction, ${ }^{1} \mathrm{H},{ }^{13} \mathrm{C}$, and HSQC NMR spectroscopies and electrospray mass spectrometry. The bond lengths and bond angles of the zinc complexes correlate well to those in horse LADH. The zinc complexes feature SNS donor atoms and pseudotetrahedral geometry about the zinc center, as is seen for liver alcohol dehydrogenase. The SNS ligand precursors were characterized with ${ }^{1} \mathrm{H},{ }^{13} \mathrm{C}$, and HSQC NMR spectroscopies and cyclic voltammetry, and were found to be redox active. Gaussian calculations were performed and agree quite well with the experimentally observed oxidation potential for the pincer ligand. The zinc complexes were screened for the reduction of electron poor aldehydes in the presence of a hydrogen donor, 1-benzyl-1,4-dihydronicotinamide (BNAH). The zinc complexes enhance the reduction of electron poor aldehydes. Density functional theory calculations were performed to better understand why the geometry about the zinc center is pseudotetrahedral rather than pseudo-square planar, which is seen for most pincer complexes. For the SNS tridentate pincer complexes, the data indicate that the pseudo-tetrahedral geometry was $43.8 \mathrm{kcal} / \mathrm{mol}$ more stable than the pseudo-square planar geometry. Density functional theory calculations were also performed on zinc complexes with monodentate ligands and the data indicate that the pseudo-tetrahedral geometry was 30.6 $\mathrm{kcal} / \mathrm{mol}$ more stable than pseudo-square planar geometry. Overall, the relative stabilities of the pseudo-tetrahedral and pseudo-square planar systems are the same for this
\end{abstract}


coordination environment whether the ligand set is a single tridentate SNS system or is

broken into three separate units. The preference of a $\mathrm{d}^{10} \mathrm{Zn}$ center to attain a tetrahedral local environment trumps any stabilization gained by removal of constraints within the ligand set.

\section{Keywords:}

SNS pincer ligand

Mononuclear Zn complexes

X-ray crystallography

Cyclic voltammetry

Density functional theory calculations

Aldehyde reductions

\section{Introduction:}

Bioinorganic chemists have made contributions toward the understanding of enzymatic activity through the synthesis and subsequent structural and functional characterization of model complexes for metalloenzyme active sites [1]. Model complexes are low molecular mass systems that seek to mimic the metalloenzyme in terms of ligand donor atoms, structure, and oxidation states [2]. Bio-inspired model complexes can be prepared to investigate the structure and function of enzymes through the use of Nature as a model for the design of highly active and efficient catalysts.

Liver alcohol dehydrogenase $(\mathrm{LADH})$ is a zinc metalloenzyme that catalyzes the oxidation of alcohols to aldehydes and ketones, and also catalyzes the reverse reaction: the reduction of a ketone or an aldehyde to an alcohol $[1,3]$. The crystal structure of horse LADH has been solved [4]. The resting enzyme has a zinc(II) metal center which is pseudo-tetrahedrally ligated with one N-histidine side chain, two S-cysteine side chains, 
and one labile water molecule. The nitrogen and sulfur donor atoms are provided by histidine and cysteine residues of a single polypeptide chain [5]. Several structural models for LADH have been reported with the same donor atoms as the metalloenzyme [6-13]. In some cases, reactivity data was not reported [14]. Reactivity has also been reported for zinc LADH model complexes with donor atoms that are different than the enzyme's active site [15].

In our efforts to understand the catalytic activity of such a metalloenzyme, we have chosen to model the structure and reactivity of the zinc active site through the use of a new family of robust pincer ligands that provide the same S,N,S donor atoms as the enzyme. Tridentate pincer ligands, first published in 1976, offer several advantages as compared to monodentate ligands. First, their metallation is favored due to a less negative delta entropy of formation as compared to monodentate ligands, conferring added stability to their metal complexes [16]. Second, tridentate pincer ligands are known to inhibit dimerization, a process that could slow or inhibit catalytic activity. Third, the structural and electronic properties of the tridentate ligand can be systematically changed by the use of slightly different materials in their synthetic preparation. The tridentate pincer ligand usually coordinates to the metal in a meridional fashion. Sometimes both of the metallocycles pucker, leading to a pseudo-facial coordination mode.

Pincer ligands have been utilized successfully in organometallic chemistry to prepare highly catalytically active and robust complexes [17]. Interestingly, to the best of our knowledge, such ligand systems have not been used frequently in bio-inspired modeling chemistry. The pincer ligand is an excellent one to model biological activity since the N- 
donor pyridine, like N-histidine, is $\mathrm{sp}^{2}$-hybridized and thioimidazolyl sulfur donors have been reported by Parkin and Vahrenkamp to model thiol derived ligands in bio-inspired zinc chemistry $[18,19]$.

Toward the goal of synthesizing and understanding bio-inspired model complexes for the liver alcohol dehydrogenase active site, we report herein the syntheses, spectroscopic and electrochemical characterization, density functional theory (DFT) calculations and activity screening of tridentate SNS zinc pincer complexes.

\section{Experimental:}

\subsection{General Procedures:}

All reagents used are commercially available and were used as received. Isopropyl imidazole, neopentyl imidazole, 2,6-bis(3-butylimidazol-1-yl)pyridine bromide, 2,6-bis(3-isopropylimidazol-1-yl)pyridine bromide, and 2,6-bis(3isopropylimidazol-2-thione-1-yl)pyridine were prepared as reported previously [20-24] as were $\mathrm{BNA}^{+}$and $\mathrm{BNAH}[25]$. NMR spectra were recorded at $25^{\circ} \mathrm{C}$ on a Bruker Avance $300 \mathrm{MHz}$ NMR spectrometer. Spectra were referred to the solvent residual peak. Electrospray mass spectrometry was performed on a Micromass ZQ instrument using nitrogen as the drying and nebulizing gas. Cyclic voltammetry experiments were performed using a Cypress Electroanalytical System with a silver wire reference electrode, a glassy carbon working electrode, and a platinum counter electrode. The supporting electrolyte for the cyclic voltammetry experiments was tetra-Nbutylammonium tetrafluoroborate. The ferrocenium/ferrocene couple was used as an internal reference; reduction potential values were corrected by assigning the 
ferrocenium/ferrocene couple to $0.40 \mathrm{~V}$ versus SCE. When an inert atmosphere was needed, a M-Braun inert atmosphere glove box and standard Schlenk techniques were used with thoroughly degassed solvents. IR spectra were collected using a Thermo Nicolet AVATAR 380-FT-IR with a SMART SPECULATR reflectance adaptor. C, H, $\mathrm{N}$ elemental analyses were performed by Atlantic Microlab Inc. (Norcross, GA). Melting points are uncorrected.

\subsection{Crystallographic Analyses:}

Crystals of $\mathbf{1}, \mathbf{2}$, and $\mathbf{3}$ were mounted on a glass fiber or loop and placed in a -80 ${ }^{\circ} \mathrm{C}$ nitrogen stream on a Bruker diffractometer equipped with a Smart CCD at Boston College (Chestnut Hill, MA). Crystallographic data were collected using graphite monochromated $0.71073 \AA$ Mo-K $\alpha$ radiation and integrated and corrected for absorption using the Bruker SAINTPLUS software package. The structures were solved using direct methods and refined using least-square methods on F-squared. All other pertinent crystallographic details such as $\mathrm{h}, \mathrm{k}, 1$ ranges, $2 \theta$ ranges, and $\mathrm{R}$-factors can be found in table 1 . 
Table 1. Crystal and Structure Refinement Data for 1-3

\begin{tabular}{|c|c|c|c|}
\hline & $\mathrm{R}=i \operatorname{Pr}(\mathbf{1})$ & $R=N p(2)$ & $\mathrm{R}=n \mathrm{Bu}(\mathbf{3})$ \\
\hline Formula & $\mathrm{C}_{17} \mathrm{H}_{22} \mathrm{Cl}_{4} \mathrm{~N}_{5} \mathrm{OS}_{2} \mathrm{Zn}_{2}$ & $\mathrm{C}_{21} \mathrm{H}_{30} \mathrm{Cl}_{4} \mathrm{~N}_{5} \mathrm{OS}_{2} \mathrm{Zn}_{2}$ & $\mathrm{C}_{19} \mathrm{H}_{25} \mathrm{Cl}_{4} \mathrm{~N}_{5} \mathrm{~S}_{2} \mathrm{Zn}_{2}$ \\
\hline FW (g/mol) & 649.06 & 705.16 & 660.10 \\
\hline Temperature (K) & 193(2) & $193(2)$ & 193(2) \\
\hline Wavelength $(\AA)$ & 0.71073 & 0.71073 & 0.71073 \\
\hline Crystal System & Monoclinic & Triclinic & Monoclinic \\
\hline Space Group & $\mathrm{P} 2(1) / \mathrm{c}$ & P-1 & $\mathrm{P} 2(1) / \mathrm{c}$ \\
\hline $\mathrm{a}(\AA)$ & $7.475(2)$ & $7.6011(19)$ & $16.5135(6)$ \\
\hline $\mathrm{b}(\AA)$ & $27.456(8)$ & 13.297(3) & $12.5308(5)$ \\
\hline c $(\AA)$ & $12.705(3)$ & $14.952(4)$ & $12.7522(5)$ \\
\hline$\alpha\left(^{\circ}\right)$ & 90 & $96.534(5)$ & 90 \\
\hline$\beta\left(^{\circ}\right)$ & $98.163(6)$ & $97.139(5)$ & $94.713(2)$ \\
\hline$\gamma\left({ }^{\circ}\right)$ & 90 & $102.721(5)$ & 90 \\
\hline Volume $(\AA)^{3}$ & $2580.9(12)$ & 1447.1(6) & $2629.86(18)$ \\
\hline Z & 4 & 2 & 4 \\
\hline $\mathrm{r}($ calc $)\left(\mathrm{g} / \mathrm{cm}^{3}\right)$ & 1.670 & 1.618 & 1.667 \\
\hline $\operatorname{Abs}\left(\mathrm{mm}^{-1}\right)$ & 2.454 & 2.196 & 2.407 \\
\hline$F(000)$ & 1308 & 718 & 1336 \\
\hline Crystal Size $\left(\mathrm{mm}^{3}\right)$ & $0.10 \times 0.02 \times 0.02$ & $0.10 \times 0.05 \times 0.02$ & $0.12 \times 0.02 \times 0.02$ \\
\hline Theta Range $\left({ }^{\circ}\right)$ & 1.48 to 28.36 & 1.39 to 28.39 & 1.24 to 27.57 \\
\hline Refl/Uniq & $31809 / 6362$ & $18174 / 6979$ & $23665 / 6059$ \\
\hline $\mathrm{R}$ (int) & 0.1605 & 0.0692 & 0.0398 \\
\hline Abs Correction & None & None & None \\
\hline Max./Min. & $0.9526 / 0.7914$ & $0.9574 / 0.8103$ & $0.9534 / 0.7610$ \\
\hline Ref Method & $\begin{array}{l}\text { Full Matrix least } \\
\text { squares on } F^{2}\end{array}$ & $\begin{array}{l}\text { Full Matrix least } \\
\text { squares on } \mathrm{F}^{2}\end{array}$ & $\begin{array}{l}\text { Full Matrix least } \\
\text { squares on } \mathrm{F}^{2}\end{array}$ \\
\hline Data / restr / par & $6362 / 0 / 286$ & $6979 / 0 / 323$ & $6059 / 0 / 291$ \\
\hline GOF on $\mathrm{F}^{2}$ & 1.122 & 1.092 & 0.790 \\
\hline $\mathrm{R} 1$ indices $(\mathrm{I}>2 \mathrm{~s})$ & 0.0884 & 0.0636 & 0.0333 \\
\hline wR2 & 0.1577 & 0.1376 & 0.0806 \\
\hline Peak/hole $\left(\mathrm{e} / \AA^{-3}\right)$ & 0.821 and -0.959 & 0.835 and -0.742 & 0.577 and -0.382 \\
\hline
\end{tabular}

\subsection{Reactivity:}

In a typical reaction, $0.1 \mathrm{mmol}$ of 4-nitrobenzaldehyde, $0.2 \mathrm{mmol}$ of $\mathrm{BNAH}$, and $0.1 \mathrm{mmol}$ of the zinc complex or $0.2 \mathrm{mmol} \mathrm{ZnCl}_{2}$ were dissolved in $3 \mathrm{~mL}$ of $\mathrm{CDCl}_{3}$. The reaction was heated at reflux. Aliquots of the reaction were taken at certain times and analyzed using ${ }^{1} \mathrm{H}$ NMR spectroscopy. All data are averages of at least two runs. 


\subsection{Gaussian Calculations:}

Gaussian 03 was used to perform single-point calculations and DFT geometry optimizations using the B3LYP hybrid functional with $6-31 \mathrm{G}^{*}$ basis sets as provided with the software [26]. Calculations were performed on the pincer ligand precursor alone as well as on the bound $\mathrm{Zn}(\mathrm{II})$ system $(\mathrm{R}=\mathrm{Me}$ in all cases). Symmetry was imposed on the metal-ligated complex such that one pseudo-tetrahedral $\left(\mathrm{C}_{\mathrm{s}}\right)$ and two pseudo-square planar $\left(\mathrm{C}_{2}\right.$ and $\left.\mathrm{C}_{2 \mathrm{v}}\right)$ structures were examined. In accordance with the molecular point groups, the SNS ligand set was required to be flat under $\mathrm{C}_{2 \mathrm{v}}$ symmetry, but could become nonplanar under $\mathrm{C}_{2}$ or $\mathrm{C}_{\mathrm{s}}$ symmetries. Frequency analysis was performed on the optimized structures to determine whether or not they represented true minima. We found no imaginary frequencies for the pseudo-tetrahedral structures, but we did find several for the pseudo-square planar systems as indicated in the discussion that follows.

Gas-phase geometry optimizations and single-point calculations using a solvent model were also performed on the unbound pincer ligand precursor in an effort to model the oxidation potentials that have been determined experimentally. After the structures of the ligand precursor were optimized in neutral and singly cationic and anionic states under each of the point groups described above, single-point SCRF calculations using DMSO via the CPCM solvent model were performed. The "radii=uff" and "nosymmcav" directives were employed. Oxidation and reduction potentials were then determined by finding the difference in the total free energies in solution for the neutral and cationic or anionic species. These $\Delta \mathrm{G}$ values were then referenced to the absolute SCE potential in DMSO by subtracting 3.83V (the established correction [27] to SHE in DMSO) and 0.241 V (the difference between SHE and SCE). 


\subsection{Syntheses:}

\subsubsection{Preparation of 2,6-bis(3-neopentylimidazol-1-yl)pyridine bromide}

$\left(\mathrm{C}_{21} \mathrm{H}_{31} \mathrm{~N}_{5} \mathrm{Br}_{2}\right)[\mathbf{2 a}]:$

In a round-bottom flask, $3.57 \mathrm{~g}(25.8 \mathrm{mmol})$ of neopentyl imidazole were added along with $3.20 \mathrm{~g}(13.5 \mathrm{mmol})$ of 2,6-dibromopyridine. The solution was brown and oily. The reaction mixture was heated neat at $160^{\circ} \mathrm{C}$ for 18 hours. After the reaction mixture was removed from the heat, the dark brown solid that remained in the round-bottom flask was dissolved in methanol $(15 \mathrm{~mL})$ the product was precipitated out of solution with $125 \mathrm{~mL}$ of diethyl ether. The tan crystals were collected by filtration through a Buchner funnel and were allowed to air dry. The yield was $3.07 \mathrm{~g}(44.3 \%)$. Melting point: $317-320{ }^{\circ} \mathrm{C}$ (dec). Anal calc. for $\mathrm{C}_{21} \mathrm{H}_{31} \mathrm{~N}_{5} \mathrm{Br}_{2} \cdot \mathrm{H}_{2} \mathrm{O}$ (531.33): C, 47.47; H, 6.26; N, 13.18. Found: C, 47.38; H, 6.23; N, 13.08 .

${ }^{1} \mathrm{H}$ NMR (DMSO-d $\left.6,300 \mathrm{MHz}\right) \delta 10.85$ ppm (s, 2H, imidazole CH), 8.93 ppm (s, 2H, imidazole $\mathrm{CH}), 8.62 \mathrm{ppm}(\mathrm{m}, 1 \mathrm{H}$, pyridine $\mathrm{CH}), 8.34 \mathrm{ppm}\left(\mathrm{d}\left({ }^{3} \mathrm{~J}=8.1 \mathrm{~Hz}\right), 2 \mathrm{H}\right.$, pyridine $\mathrm{CH}), 8.10 \mathrm{ppm}(\mathrm{s}, 2 \mathrm{H}$, imidazole $\mathrm{CH}), 4.25 \mathrm{ppm}\left(\mathrm{s}, 2 \mathrm{H}, \mathrm{CH}_{2}\right), 1.02 \mathrm{ppm}\left(\mathrm{s}, 18 \mathrm{H}, \mathrm{CH}_{3}\right)$. ${ }^{13} \mathrm{C}\left\{{ }^{1} \mathrm{H}\right\}$ NMR (DMSO-d $\left.\mathrm{d}_{6}, 75 \mathrm{MHz}\right) \delta 145.28 \mathrm{ppm}$ (pyridine $\mathrm{CH}$ ), $144.64 \mathrm{ppm}$ (pyridine C), 136.23 ppm (imidazole $\mathrm{CH}$ ), 125.14 ppm (imidazole $\mathrm{CH}$ ), 119.11 ppm (imidazole $\mathrm{CH}), 114.29$ ppm (pyridine $\mathrm{CH}), 59.99$ ppm $\left(\mathrm{CH}_{2}\right), 32.08\left(\mathrm{C}\left(\mathrm{CH}_{3}\right)_{3}\right), 26.69 \mathrm{ppm}\left(\mathrm{CH}_{3}\right)$.

\subsubsection{Preparation of 2,6-bis(3-neopentylimidazol-2-thione-1-yl)pyridine,} $\left(\mathrm{C}_{21} \mathrm{H}_{29} \mathrm{~N}_{5} \mathrm{~S}_{2}\right)[\mathbf{2 b}]:$

A $100 \mathrm{~mL}$ round-bottom flask was charged with $0.52 \mathrm{~g}(1.0 \mathrm{mmol}) 2,6-\mathrm{bis}(3-$ neopentylimidazol-1-yl)pyridine bromide, $0.20 \mathrm{~g}(2.4 \mathrm{mmol})$ sodium acetate and $40 \mathrm{~mL}$ 
of acetonitrile. The solution was stirred at reflux for 30 minutes after which not all of the sodium acetate had dissolved. Then $0.66 \mathrm{~g}(21 \mathrm{mmol})$ sulfur was added to the roundbottom flask with an additional $10 \mathrm{~mL}$ of acetonitrile. The solution was stirred at reflux for three days. The product was vacuum filtered to remove the remaining solids. The solvent was removed under reduced pressure. The yield $(0.48 \mathrm{~g})$ was quantitative. Electrospray MS (MeOH, 20V, positive ion mode (m/z): Expected: $415.2(100 \%)$ $\left[\mathrm{C}_{21} \mathrm{H}_{29} \mathrm{~N}_{5} \mathrm{~S}_{2}\right]^{+}$, Found: $416.5(100 \%)\left[\mathrm{C}_{21} \mathrm{H}_{29} \mathrm{~N}_{5} \mathrm{~S}_{2}+1\right]^{+}$. Anal calc. for $\mathrm{C}_{21} \mathrm{H}_{29} \mathrm{~N}_{5} \mathrm{~S}_{2} \cdot \mathrm{H}_{2} \mathrm{O}$ (415.62): C, 58.17; H, 7.21; N, 16.15. Found: C, 57.58; H, 6.72; N, 15.80.

${ }^{1} \mathrm{H}$ NMR $\left(\right.$ DMSO-d $\left._{6}, 300 \mathrm{MHz}\right) \delta 8.66 \mathrm{ppm}\left(\mathrm{d}\left({ }^{3} \mathrm{~J}=8.1 \mathrm{~Hz}\right), 2 \mathrm{H}\right.$, pyridine $\left.\mathrm{CH}\right), 8.19 \mathrm{ppm}$ ( $\mathrm{t}\left({ }^{3} \mathrm{~J}=8.1 \mathrm{~Hz}\right), 1 \mathrm{H}$, pyridine $\left.\mathrm{CH}\right), 7.83 \mathrm{ppm}\left(\mathrm{d}\left({ }^{3} \mathrm{~J}=2.4 \mathrm{~Hz}\right), 2 \mathrm{H}\right.$, imidazole $\left.\mathrm{CH}\right), 7.33$ ppm $\left(\mathrm{d}\left({ }^{3} \mathrm{~J}=2.7 \mathrm{~Hz}\right), 2 \mathrm{H}\right.$, imidazole $\left.\mathrm{CH}\right), 4.00 \mathrm{ppm}\left(\mathrm{s}, 4 \mathrm{H}, \mathrm{CH}_{2}\right), 1.00 \mathrm{ppm}\left(\mathrm{s}, 18 \mathrm{H}, \mathrm{CH}_{3}\right)$, ${ }^{13} \mathrm{C}\left\{{ }^{1} \mathrm{H}\right\}$ NMR $\left(\mathrm{DMSO}-\mathrm{d}_{6}, 75 \mathrm{MHz}\right) \delta 163.07 \mathrm{ppm}(\mathrm{C}=\mathrm{S}), 148.43$ ppm (pyridine C), 139.77 ppm (pyridine $\mathrm{CH}$ ), 119.99 ppm (imidazole $\mathrm{CH}$ ), 117.35 ppm (pyridine $\mathrm{CH}$ ), 116.19 ppm (imidazole $\mathrm{CH}), 56.58$ ppm $\left(\mathrm{CH}_{2}\right), 33.75\left(\mathrm{C}\left(\mathrm{CH}_{3}\right)_{3}\right), 27.86 \mathrm{ppm}\left(\mathrm{CH}_{3}\right)$. Selected IR bands (reflectance): $v_{\max } / \mathrm{cm}^{-1} 1125(\mathrm{C}=\mathrm{S})$.

2.5.3 Preparation of 2,6-bis[3-(n-butyl)imidazol-2-thione-1-yl]pyridine, $\left(\mathrm{C}_{19} \mathrm{H}_{25} \mathrm{~N}_{5} \mathrm{~S}_{2}\right)$ : $[3 \mathbf{b}]$

A round-bottom flask was charged with $0.48 \mathrm{~g}(1.0 \mathrm{mmol})$ of $2,6-\mathrm{bis}[3-(n-$ butyl)imidazol-1-yl]pyridine bromide, $0.24 \mathrm{~g}(3.0 \mathrm{mmol})$ of sodium acetate and $40 \mathrm{~mL}$ of $\mathrm{MeCN}$. The mixture was heated at reflux. After 30 minutes, $0.68 \mathrm{~g}(21 \mathrm{mmol})$ of sulfur was added. The reaction was heated at reflux for seven days. The product was vacuum filtered to remove the remaining solids. The solvent was removed under reduced 
pressure. The yield was $0.38 \mathrm{~g}$ (70. \%). Electrospray MS (MeOH, 20V, positive ion mode (m/z): Expected: $387.2(100 \%)\left[\mathrm{C}_{19} \mathrm{H}_{25} \mathrm{~N}_{5} \mathrm{~S}_{2}\right]^{+}$, Found: $388.6(100 \%)\left[\mathrm{C}_{19} \mathrm{H}_{25} \mathrm{~N}_{5} \mathrm{~S}_{2}\right.$ $+1]^{+}$. Anal calc. for $\mathrm{C}_{19} \mathrm{H}_{25} \mathrm{~N}_{5} \mathrm{~S}_{2} \cdot 1.5 \mathrm{H}_{2} \mathrm{O}$ (387.57): C, 55.04; H, 6.81; N, 16.89. Found: C, 55.22; H, 6.26; N, 16.73 .

${ }^{1} \mathrm{H}$ NMR (DMSO-d 6 , $\left.300 \mathrm{MHz}\right) \delta 8.68 \mathrm{ppm}\left(\mathrm{d}\left({ }^{3} \mathrm{~J}=7.8 \mathrm{~Hz}\right), 2 \mathrm{H}\right.$, pyridine $\left.\mathrm{CH}\right), 8.20 \mathrm{ppm}$ $\left(\mathrm{t}\left({ }^{3} \mathrm{~J}=8.1 \mathrm{~Hz}\right), 1 \mathrm{H}\right.$, pyridine $\left.\mathrm{CH}\right), 7.82 \mathrm{ppm}\left(\mathrm{d}\left({ }^{3} \mathrm{~J}=2.4 \mathrm{~Hz}\right), 2 \mathrm{H}\right.$, imidazole $\left.\mathrm{CH}\right), 7.42$ ppm (d ( $\left.{ }^{3} \mathrm{~J}=2.7 \mathrm{~Hz}\right), 2 \mathrm{H}$, imidazole $\left.\mathrm{CH}\right), 4.05 \mathrm{ppm}\left(\mathrm{t}\left({ }^{3} \mathrm{~J}=7.6 \mathrm{~Hz}\right), 4 \mathrm{H}, \mathrm{CH}_{2}\right), 1.73 \mathrm{ppm}$ (m, 4H, CH$), 1.33 \mathrm{ppm}\left(\mathrm{m}, 4 \mathrm{H}, \mathrm{CH}_{2}\right), 0.93 \mathrm{ppm}\left(\mathrm{t}\left({ }^{3} \mathrm{~J}=7.5 \mathrm{~Hz}\right), 6 \mathrm{H}, \mathrm{CH}_{3}\right) .{ }^{13} \mathrm{C}\left\{{ }^{1} \mathrm{H}\right\}$ NMR (DMSO-d 6 , 75MHz) $\delta 161.26$ ppm (C=S), 148.27 ppm (pyridine C), 139.90 ppm (pyridine $\mathrm{CH}$ ), 118.86 ppm (imidazole $\mathrm{CH}$ ), 116.99 ppm (pyridine $\mathrm{CH}$ ), $116.47 \mathrm{ppm}$ (imidazole $\mathrm{CH}), 46.47 \mathrm{ppm}\left(\mathrm{CH}_{2}\right), 29.96\left(\mathrm{CH}_{2}\right), 19.19\left(\mathrm{CH}_{2}\right), 13.54 \mathrm{ppm}\left(\mathrm{CH}_{3}\right)$. Selected IR bands (reflectance): $v_{\max } / \mathrm{cm}^{-1} 1126(\mathrm{C}=\mathrm{S})$.

\subsubsection{Preparation of chloro $\left(\eta^{3}-\mathrm{S}, \mathrm{S}, \mathrm{N}\right)-[2,6$-bis(3-isopropylimidazol-2-thione-1-} yl)]pyridinezinc(II) aquatrichlorozincate, $\left(\left[\mathrm{C}_{17} \mathrm{H}_{21} \mathrm{~N}_{5} \mathrm{~S}_{2} \mathrm{ZnCl}\right]\left[\mathrm{ZnCl}_{3}\left(\mathrm{OH}_{2}\right)\right]\right)$ : [1]

In a $100 \mathrm{~mL}$ round-bottom flask, $0.062 \mathrm{~g}(0.17 \mathrm{mmol}) 2,6$-bis(3-isopropylimidazol2-thione-1-yl)pyridine was combined with $0.057 \mathrm{~g}(0.42 \mathrm{mmol}) \mathrm{ZnCl}_{2}$ and dissolved in $10 \mathrm{~mL} \mathrm{CH}_{2} \mathrm{Cl}_{2}$. The solution was stirred at reflux for $18 \mathrm{~h}$ and the off-white solid was collected by vacuum filtration. The yield for the reaction was $0.070 \mathrm{~g}(63 \%)$. Single crystals for X-ray diffraction were grown by a slow vapor diffusion of diethyl ether into a dichloromethane solution containing the zinc complex. Electrospray MS (MeOH, 0 V, positive ion mode (m/z)): Expected: $460.0(99 \%)\left[C_{17} H_{21} N_{5} S_{2} \mathrm{ZnCl}\right]^{+}$, Found: 459.9 
(100\%) $\left[C_{17} H_{2 I} N_{5} S_{2} \mathrm{ZnCl}\right]^{+}$. Anal calc. for $\mathrm{C}_{17} \mathrm{H}_{23} \mathrm{Cl}_{4} \mathrm{~N}_{5} \mathrm{OS}_{2} \mathrm{Zn}_{2} \cdot \mathrm{MeOH} \cdot 2 \mathrm{H}_{2} \mathrm{O}$ (718.17):

C, 30.10; H, 4.35; N, 9.75. Found: C, 30.00; H, 3.91; N, 9.48.

${ }^{1} \mathrm{H}$ NMR (DMSO-d $\left.\mathrm{d}_{6}, 300 \mathrm{MHz}\right) \delta 8.64 \mathrm{ppm}\left(\mathrm{d}\left({ }^{3} \mathrm{~J}=8.1 \mathrm{~Hz}\right), 2 \mathrm{H}\right.$, pyridine $\left.\mathrm{CH}\right), 8.19 \mathrm{ppm}$ $\left(\mathrm{t}\left({ }^{3} \mathrm{~J}=8.1 \mathrm{~Hz}\right), 1 \mathrm{H}\right.$, pyridine $\left.\mathrm{CH}\right), 7.84 \mathrm{ppm}\left(\mathrm{d}\left({ }^{3} \mathrm{~J}=2.7 \mathrm{~Hz}\right), 2 \mathrm{H}\right.$, imidazole $\left.\mathrm{CH}\right), 7.52$ ppm (d ( $\left.{ }^{3} \mathrm{~J}=2.7 \mathrm{~Hz}\right), 2 \mathrm{H}$, imidazole $\left.\mathrm{CH}\right), 5.02 \mathrm{ppm}(\mathrm{m}, 2 \mathrm{H}$, isopropyl $\mathrm{CH}), 1.35 \mathrm{ppm}(\mathrm{d}$ $\left({ }^{3} \mathrm{~J}=6.6 \mathrm{~Hz}\right), 12 \mathrm{H}$, isopropyl $\left.\mathrm{CH}_{3}\right) .{ }^{13} \mathrm{C}\left\{{ }^{1} \mathrm{H}\right\}$ NMR (DMSO-d 6 , 75MHz) $\delta 160.53 \mathrm{ppm}$ $(\mathrm{C}=\mathrm{S}), 148.16 \mathrm{ppm}$ (pyridine $\mathrm{C}$ ), $140.00 \mathrm{ppm}$ (pyridine $\mathrm{CH}$ ), 117.48 ppm (imidazole $\mathrm{CH}), 117.17$ ppm (pyridine $\mathrm{CH}$ ), 115.25 ppm (imidazole $\mathrm{CH}$ ), 48.12 ppm (isopropyl $\mathrm{CH}$ ), 21.00 ppm (isopropyl $\mathrm{CH}_{3}$ ).

${ }^{1} \mathrm{H}$ NMR (MeOD-d $\left.3,300 \mathrm{MHz}\right) \delta 8.72 \mathrm{ppm}\left(\mathrm{d}\left({ }^{3} \mathrm{~J}=8.1 \mathrm{~Hz}\right), 2 \mathrm{H}\right), 8.15 \mathrm{ppm}(\mathrm{m}, 1 \mathrm{H}), 7.70$ ppm $\left(\mathrm{d}\left({ }^{3} \mathrm{~J}=2.7 \mathrm{~Hz}\right), 2 \mathrm{H}\right), 7.33 \mathrm{ppm}\left(\mathrm{d}\left({ }^{3} \mathrm{~J}=2.7 \mathrm{~Hz}\right), 2 \mathrm{H}\right), 5.21 \mathrm{ppm}(\mathrm{m}, 2 \mathrm{H}$, isopropyl $\mathrm{CH}), 1.19 \mathrm{ppm}\left(\mathrm{d}\left({ }^{3} \mathrm{~J}=7.2 \mathrm{~Hz}\right), 12 \mathrm{H}\right.$, isopropyl $\left.\mathrm{CH}_{3}\right)$.

\subsubsection{Preparation of chloro $\left(\eta^{3}-\mathrm{S}, \mathrm{S}, \mathrm{N}\right)-[2,6-b i s(3-n e o p e n t y l i m i d a z o l-2-t h i o n e-1-$ yl)]pyridinezinc(II) aquatrichlorozincate $\left[\mathrm{C}_{21} \mathrm{H}_{27} \mathrm{~N}_{5} \mathrm{~S}_{2} \mathrm{ZnCl}\right]\left[\mathrm{ZnCl}_{3}\left(\mathrm{OH}_{2}\right)\right]$ : [2]}

In a round-bottom flask, $0.14 \mathrm{~g}(0.35 \mathrm{mmol})$ of 2,6-bis(3-neopentylimidazol-2-thione-1yl)pyridine was added to $0.10 \mathrm{~g}(0.73 \mathrm{mmol})$ of $\mathrm{ZnCl}_{2}$. The reactants were dissolved in 8 $\mathrm{mL}$ of dichloromethane. The yellow solution was heated at reflux for 18 hours. An offwhite precipitate was present at the end of the reaction. The solvent was removed under reduced pressure. Single crystals for X-ray diffraction were grown by a slow vapor diffusion of pentane into a dichloromethane solution containing the zinc complex. The yield for the synthesis was $0.24 \mathrm{~g}$ (quantitative). Electrospray MS (MeOH, $0 \mathrm{~V}$, positive ion mode (m/z): Expected: $514.0(100 \%)\left[C_{21} H_{27} N_{5} S_{2} \mathrm{ZnCl}\right]^{+}$, Found: $514.3(100 \%)$ 
$\left[\mathrm{C}_{21} \mathrm{H}_{27} \mathrm{~N}_{5} \mathrm{~S}_{2} \mathrm{ZnCl}\right]^{+}$. Anal calc. for $\mathrm{C}_{21} \mathrm{H}_{31} \mathrm{Cl}_{4} \mathrm{~N}_{5} \mathrm{OS}_{2} \mathrm{Zn}_{2} \cdot \mathrm{CH}_{3} \mathrm{CN} \cdot \mathrm{Et}_{2} \mathrm{O}$ (706.21): $\mathrm{C}$, 39.48; H, 5.40; N, 10.23. Found: C, 39.21; H, 5.21; N, 10.75 .

${ }^{1} \mathrm{H}$ NMR (DMSO-d 6 , $\left.300 \mathrm{MHz}\right) \delta 8.66 \mathrm{ppm}\left(\mathrm{d}\left({ }^{3} \mathrm{~J}=8.1 \mathrm{~Hz}\right), 2 \mathrm{H}\right.$, pyridine $\left.\mathrm{CH}\right), 8.19$ ppm ( $\left(\left(^{3} \mathrm{~J}=8.1 \mathrm{~Hz}\right), 1 \mathrm{H}\right.$, pyridine $\left.\mathrm{CH}\right), 7.83 \mathrm{ppm}\left(\mathrm{d}\left({ }^{3} \mathrm{~J}=2.4 \mathrm{~Hz}\right), 2 \mathrm{H}\right.$, imidazole $\left.\mathrm{CH}\right)$, 7.33 ppm (d ( $\left.{ }^{3} \mathrm{~J}=2.1 \mathrm{~Hz}\right), 2 \mathrm{H}$, pyridine $\left.\mathrm{CH}\right), 4.00 \mathrm{ppm}\left(\mathrm{s}, 4 \mathrm{H}, \mathrm{CH}_{2}\right), 1.00 \mathrm{ppm}(\mathrm{s}, 18 \mathrm{H}$, $\left.\mathrm{CH}_{3}\right) .{ }^{13} \mathrm{C}\left\{{ }^{1} \mathrm{H}\right\}$ NMR (DMSO-d $\left.6,75 \mathrm{MHz}\right) \delta 163.08 \mathrm{ppm}(\mathrm{C}=\mathrm{S}), 148.46 \mathrm{ppm}$ (pyridine C), 139.32 ppm (pyridine $\mathrm{CH}$ ), 120.04 ppm (imidazole $\mathrm{CH}$ ), 117.42 ppm (pyridine $\mathrm{CH}$ ),

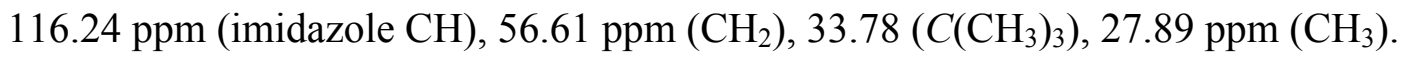
${ }^{1} \mathrm{H}$ NMR (MeOD-d $\left.{ }_{3}, 300 \mathrm{MHz}\right) \delta 8.64 \mathrm{ppm}\left(\mathrm{d}\left({ }^{3} \mathrm{~J}=8.1 \mathrm{~Hz}\right), 2 \mathrm{H}\right.$, pyridine $\left.\mathrm{CH}\right), 8.14 \mathrm{ppm}$ (m, 1H, pyridine $\mathrm{CH}), 7.69 \mathrm{ppm}\left(\mathrm{d}\left({ }^{3} \mathrm{~J}=2.4 \mathrm{~Hz}\right), 2 \mathrm{H}\right.$, imidazole $\left.\mathrm{CH}\right), 7.23 \mathrm{ppm}\left(\mathrm{d}\left({ }^{3} \mathrm{~J}\right.\right.$ $=2.1 \mathrm{~Hz}), 2 \mathrm{H}$, imidazole $\mathrm{CH}), 4.08 \mathrm{ppm}\left(\mathrm{s}, 4 \mathrm{H}, \mathrm{CH}_{2}\right), 1.05 \mathrm{ppm}\left(\mathrm{s}, 18 \mathrm{H}, \mathrm{CH}_{3}\right)$.

\subsubsection{Preparation of chloro $\left(\eta^{3}-\mathrm{S}, \mathrm{S}, \mathrm{N}\right)-\{2,6-b i s[3-(n$-butyl)imidazol-2-thione-1-} yl] \}pyridinezinc(II) aquatrichlorozincate, $\left(\left[\mathrm{C}_{19} \mathrm{H}_{25} \mathrm{~N}_{5} \mathrm{~S}_{2} \mathrm{ZnCl}\right]\left[\mathrm{ZnCl}_{3}\right]\right)$ : [3]

In a round-bottom flask, $0.15 \mathrm{~g}(0.38 \mathrm{mmol})$ of 2,6-bis[3-( $n$-butyl)imidazol-2thione-1-yl]pyridine were combined with $\mathrm{ZnCl}_{2}, 0.099 \mathrm{~g}(0.73 \mathrm{mmol})$, and dissolved in $10 \mathrm{~mL}$ of $\mathrm{CH}_{2} \mathrm{Cl}_{2}$. The solution was refluxed for $18 \mathrm{~h}$. An off-white precipitate was present at the end of the reaction. The solvent was removed under reduced pressure. Single crystals for X-ray diffraction were grown by a slow vapor diffusion of pentane into a dichloromethane solution containing the zinc complex. The yield was $0.25 \mathrm{~g}$ (quantitative). Electrospray MS (MeOH, $0 \mathrm{~V}$, positive ion mode (m/z): Expected: 486.0 (100 \%) $\left[C_{19} \mathrm{H}_{25} \mathrm{~N}_{5} \mathrm{~S}_{2} \mathrm{ZnCl}\right]^{+}$, Found: $486.4(100 \%)\left[\mathrm{C}_{19} \mathrm{H}_{25} \mathrm{~N}_{5} \mathrm{~S}_{2} \mathrm{ZnCl}\right]^{+}$. Anal calc. for 
$\mathrm{C}_{19} \mathrm{H}_{27} \mathrm{Cl}_{4} \mathrm{~N}_{5} \mathrm{OS}_{2} \mathrm{Zn}_{2} \cdot 0.5 \mathrm{CH}_{3} \mathrm{CN}$ (678.15): $\mathrm{C}, 34.38 ; \mathrm{H}, 4.11 ; \mathrm{N}, 11.03$. Found: $\mathrm{C}, 34.33$; $\mathrm{H}, 4.17 ; \mathrm{N}, 10.54$.

${ }^{1} \mathrm{H}$ NMR (DMSO-d $\left.\mathrm{d}_{6}, 300 \mathrm{MHz}\right) \delta 8.68 \mathrm{ppm}\left(\mathrm{d}\left({ }^{3} \mathrm{~J}=8.1 \mathrm{~Hz}\right), 2 \mathrm{H}\right.$, pyridine $\left.\mathrm{CH}\right), 8.20 \mathrm{ppm}$ $\left(\mathrm{t}\left({ }^{3} \mathrm{~J}=8.1 \mathrm{~Hz}\right), 1 \mathrm{H}\right.$, pyridine $\left.\mathrm{CH}\right), 7.82 \mathrm{ppm}\left(\mathrm{d}\left({ }^{3} \mathrm{~J}=2.1 \mathrm{~Hz}\right), 2 \mathrm{H}\right.$, imidazole $\left.\mathrm{CH}\right), 7.42$ ppm (d ( $\left(^{3} \mathrm{~J}=2.1 \mathrm{~Hz}\right), 2 \mathrm{H}$, imidazole $\left.\mathrm{CH}\right), 4.06 \mathrm{ppm}\left(\mathrm{t}\left({ }^{3} \mathrm{~J}=7.2 \mathrm{~Hz}\right), 4 \mathrm{H}, \mathrm{CH}_{2}\right), 1.73 \mathrm{ppm}$ (m, 4H, $\left.\mathrm{CH}_{2}\right), 1.34 \mathrm{ppm}\left(\mathrm{m}, 4 \mathrm{H}, \mathrm{CH}_{2}\right), 0.93 \mathrm{ppm}\left(\mathrm{t}\left({ }^{3} \mathrm{~J}=7.2 \mathrm{~Hz}\right), 6 \mathrm{H}, \mathrm{CH}_{3}\right) .{ }^{13} \mathrm{C}\left\{{ }^{1} \mathrm{H}\right\}$ NMR (DMSO-d 6 , 75MHz) $\delta 161.26$ ppm (C=S), 148.27 ppm (pyridine C), 139.93 ppm (pyridine $\mathrm{CH}$ ), 118.88 ppm (imidazole $\mathrm{CH}$ ), 117.04 ppm (pyridine $\mathrm{CH}$ ), $116.50 \mathrm{ppm}$ (imidazole $\mathrm{CH}), 46.48$ ppm $\left(\mathrm{CH}_{2}\right), 29.97\left(\mathrm{CH}_{2}\right), 19.19\left(\mathrm{CH}_{2}\right), 13.55 \mathrm{ppm}\left(\mathrm{CH}_{3}\right)$.

${ }^{1} \mathrm{H}$ NMR (MeOD-d $\left.3,300 \mathrm{MHz}\right) \delta 8.64 \mathrm{ppm}\left(\mathrm{d}\left({ }^{3} \mathrm{~J}=8.1 \mathrm{~Hz}\right), 2 \mathrm{H}\right.$, pyridine $\left.\mathrm{CH}\right), 8.17 \mathrm{ppm}$ (m, 1H, pyridine $\mathrm{CH}), 7.70 \mathrm{ppm}\left(\mathrm{d}\left({ }^{3} \mathrm{~J}=2.7 \mathrm{~Hz}\right), 2 \mathrm{H}\right.$, imidazole $\left.\mathrm{CH}\right), 7.26 \mathrm{ppm}\left(\mathrm{d}\left({ }^{3} \mathrm{~J}\right.\right.$ $=2.7 \mathrm{~Hz}), 2 \mathrm{H}$, imidazole $\mathrm{CH}), 4.16 \mathrm{ppm}\left(\mathrm{m}, 4 \mathrm{H}, \mathrm{CH}_{2}\right), 1.83 \mathrm{ppm}\left(\mathrm{m}, 4 \mathrm{H}, \mathrm{CH}_{2}\right), 1.42 \mathrm{ppm}$ (m, 4H, $\left.\mathrm{CH}_{2}\right), 1.03$ ppm (m, 6H, $\left.\mathrm{CH}_{3}\right)$.

\section{Results and Discussion:}

\subsection{Syntheses and Spectroscopy}

The syntheses of the tridentate SNS ligand precursors and zinc pincer complexes 1-3 were accomplished following Scheme 1. The alkyl imidazoles were either prepared following known routes or were commercially available [20,22]. Different $R$ groups were employed because Crabtree has reported that modification of such substituents affects the solubility and catalytic activity of the metal complexes [23]. The alkyl imidazoles react neatly with 2,6-dibromopyridine to form the ligand precursor salts 1a, 2a, or 3a [28], which are soluble in DMSO, methanol, acetonitrile and water. 
Compounds 1a, 2a, and 3a, react with a mild base, sodium acetate, and elemental sulfur in refluxing acetonitrile to form the bis-thione ligand precursors $\mathbf{1 b}, \mathbf{2 b}$, and $\mathbf{3 b}$, respectively [24]. Instead of sodium acetate, a stronger base, potassium t-butoxide, could also be utilized [29]. As determined by NMR spectroscopy, $\mathbf{1 b}, \mathbf{2 b}$, or $\mathbf{3 b}$ can be purified by filtering a dichloromethane solution containing this compound through alumina. These compounds are soluble in DMSO, dichloromethane, chloroform, acetone, acetonitrile, and methanol.

The bis-thione ligand precursors subsequently react with $\mathrm{ZnCl}_{2}$ in refluxing $\mathrm{CH}_{2} \mathrm{Cl}_{2}$ to afford compounds 1-3. The driving force for the metallation is the formation of zinc complexes 1-3, which are sparingly soluble in $\mathrm{CH}_{2} \mathrm{Cl}_{2}$. Off-white crystals that were suitable for x-ray diffraction were grown by allowing diethyl ether $(\mathbf{1})$ or pentane $(\mathbf{2 , 3})$ to slowly diffuse into a dichloromethane solution containing $\mathbf{1 , 2}$ or $\mathbf{3}$. All of the reactions could be carried out in air, and the reactions proceeded in $63 \%$ to quantitative yield. The zinc complexes 1-3 are soluble in dimethyl sulfoxide, acetonitrile, methanol, and water and are sparingly soluble in dichloromethane and chloroform. 



1a, $\mathrm{R}=\mathrm{iPr}$

2a, $R=$ neopentyl, $44 \%$

3a, $R=n B u$

1. $\mathrm{NaOAc}$,

$\mathrm{MeCN}$, reflux,

2. $\mathrm{S}_{8}$

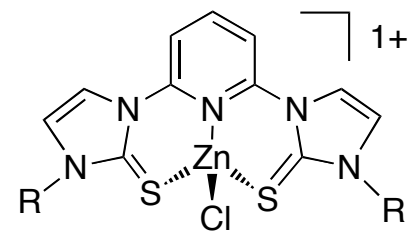

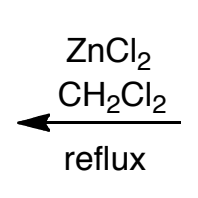<smiles>[R]n1ccn(-c2cccc(-n3ccn([R])c3=S)n2)c1=S</smiles>

1b, $\mathrm{R}=\mathrm{iPr}$

2b, $\mathrm{R}=$ neopentyl, quant.

3b, $R=n B u, 70 \%$

\begin{abstract}
1, $\mathrm{R}=\mathrm{iPr}, 63 \%$
2, $\mathrm{R}=$ neopentyl, quant.

3, $\mathrm{R}=\mathrm{nBu}$, quant.
\end{abstract}

\title{
Scheme 1: Preparation of 1-3
}

Ligand precursors 1a, 1b, 2a, 2b, 3a, and $\mathbf{3 b}$ were characterized using ${ }^{1} \mathrm{H},{ }^{13} \mathrm{C}$, and HSQC NMR spectroscopy. For these ligand precursors and zinc complexes 1-3, only one set of resonances was detected indicating that the two halves of each molecule are symmetry-related. The ${ }^{13} \mathrm{C}$ NMR spectra of $\mathbf{1 b}, \mathbf{2 b}$, and $\mathbf{3 b}$, show resonances at $\sim 161$ ppm that are consistent with $\mathrm{C}=\mathrm{S}$ formation [17]. The bis-thione ligand precursors, $\mathbf{1 b}$, $\mathbf{2 b}$, and $\mathbf{3 b}$, lacked the acidic $\mathrm{C}-\mathrm{H}$ proton at $\delta \sim 11 \mathrm{ppm}$ in the ${ }^{1} \mathrm{H}$ NMR spectra, whereas this proton resonance was present for the precursors 1a, 2a, and $\mathbf{3 a}$.

Complexes 1-3 were analyzed with ${ }^{1} \mathrm{H},{ }^{13} \mathrm{C}$, and HSQC NMR spectroscopy in DMSO$\mathrm{d}_{6}$. The ${ }^{1} \mathrm{H}$ and ${ }^{13} \mathrm{C}$ NMR spectra of the zinc complexes $\mathbf{1 - 3}$ are largely the same with very little shift in the resonances when compared to the respective ligand precursors $\mathbf{1 b}, \mathbf{2 b}$, and $\mathbf{3 b}$. This was expected since no hydrogen or carbon atoms are displaced or added upon metallation with zinc(II) chloride. In addition, the ${ }^{1} \mathrm{H}$ NMR spectra of complexes 1- 
3 were acquired in a less polar and weakly coordinating solvent, MeOD- $\mathrm{d}_{3}$, to verify that the SNS pincer ligands cannot be displaced from the coordination sphere of zinc.

Electrospray mass spectrometry data, described below, also verifies this result. Zinc complexes 1-3 are sparingly soluble in MeOD- $\mathrm{d}_{3}$. When compared to the ${ }^{1} \mathrm{H}$ NMR spectra of complexes 1-3 that were acquired in DMSO- $\mathrm{d}_{6}$, there was a slight shift $(\delta$ $\sim 0.05$ to 0.10 ) ppm of all of the resonances in the proton NMR spectra that were acquired in MeOD- $\mathrm{d}_{3}$. In the ${ }^{1} \mathrm{H}$ NMR spectra that were acquired in MeOD- $\mathrm{d}_{3}$, when compared to the ${ }^{1} \mathrm{H}$ NMR spectra acquired in DMSO- $\mathrm{d}_{6}$, some of the resonances shifted upfield while others shifted downfield.

ESI-MS spectra for compounds 1-3 were collected with cone voltages of $0 \mathrm{~V}$ and 70 V. The predominant feature in the spectra of these systems at the higher cone voltage is that of the fully ligated zinc complex, indicating that the compound is stable and suggesting that it is unlikely that the ligand is displaced when dissolved in a polar solvent. In negative ion mode, the expected $\mathrm{m} / \mathrm{z}$ values were seen for $\left[\mathrm{ZnCl}_{3}\right]^{-}$. The isotopic patterns in the mass spectrometry data fit the assigned structures.

\subsection{X-ray Crystallography}

The solid-state molecular structures of 1, 2, and $\mathbf{3}$ are shown in Figure 1. Complexes 1-3 feature SNS donor atoms and pseudotetrahedral geometry about the zinc center, as is seen for liver alcohol dehydrogenase. The bond lengths and bond angles for 1-3 are listed and are compared to the active site in horse LADH-CNAD in Table 2. CNAD is an isoteric C-glycosidic analogue of NADH containing a neutral pyridine ring, which is a potential inhibitor of LADH. During catalysis, it is proposed that cofactor and substrate 
are near the zinc(II) active site [32]. In the LADH-CNAD structure, the CNAD mimics the cofactor binding to LADH. Therefore, we selected this crystal structure because this structure contained a co-factor mimic and an ethanol molecule in the active site. The bond lengths and bond angles for 1-3 compare reasonably well to LADH-CNAD. The carbon-sulfur bond lengths of $\sim 1.7 \AA$ are between what is normally associated with a C$\mathrm{S}$ single bond, $1.83 \AA$, and a $\mathrm{C}=\mathrm{S}$ double bond, $1.61 \AA$ [30]. For 1 and 2, the counteranion is $\left[\mathrm{ZnCl}_{3}\left(\mathrm{H}_{2} \mathrm{O}\right)\right]^{-}$and for $\mathbf{3}$, the counter ion is $\left[\mathrm{ZnCl}_{3}\right]^{-}$. The counter-ion is seen even when either one or two molar equivalents of $\mathrm{ZnCl}_{2}$ are used in the preparation of 13.

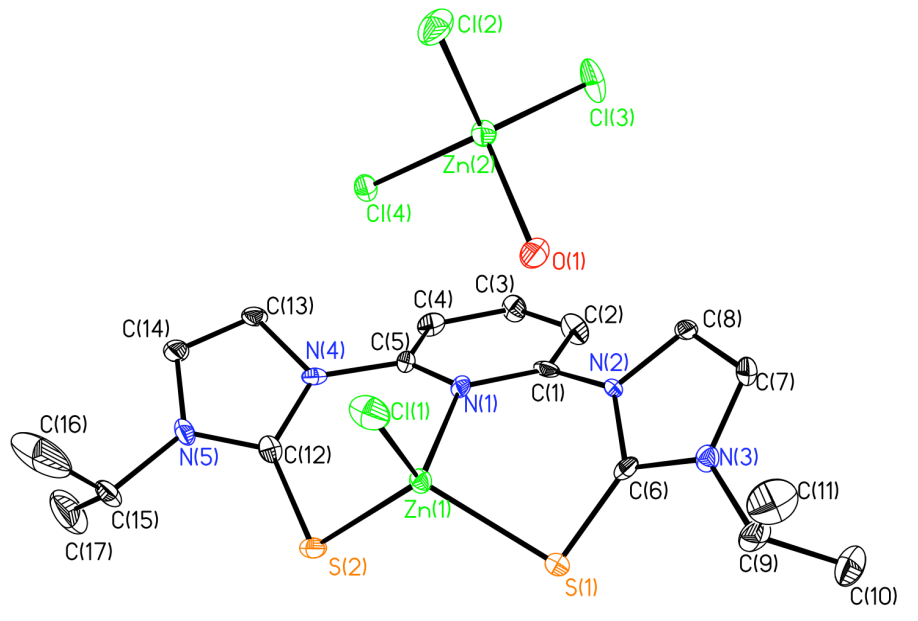

(1) 




(2)

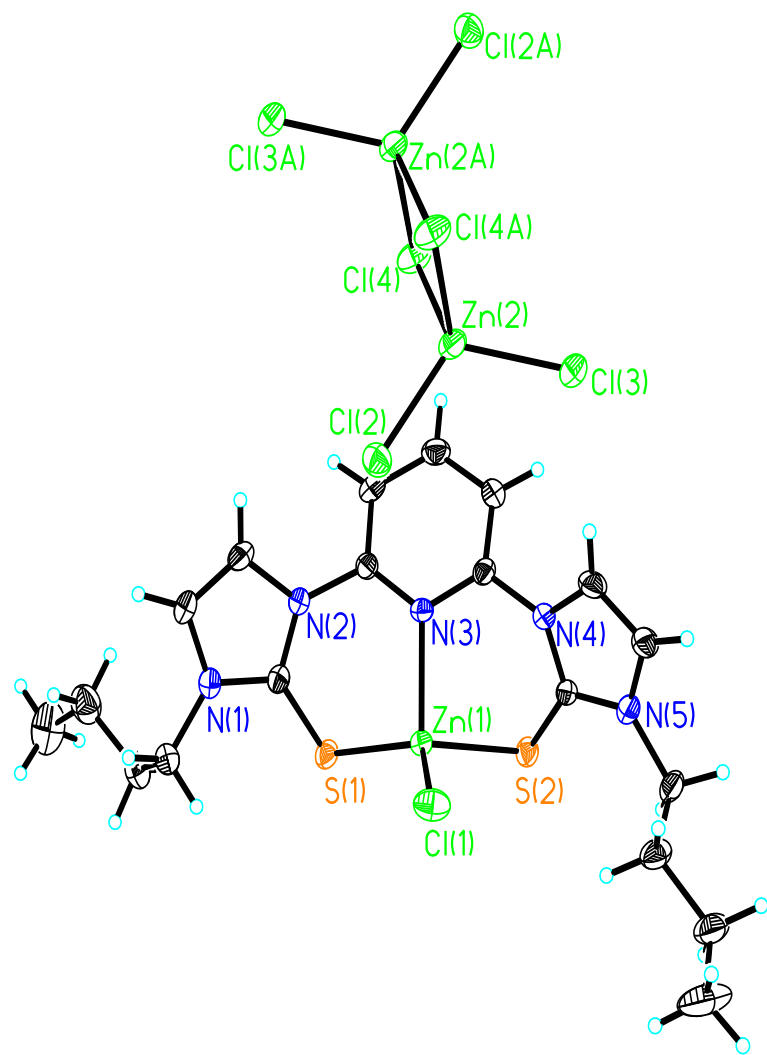

(3)

Figure 1. Solid-state structures of complexes 1-3. 
Table 2. Selected bond lengths and angles (esd) for 1-3 and comparison to LADHCNAD [31]

\begin{tabular}{|c|c|c|c|c|}
\hline & $\mathrm{R}=i \operatorname{Pr}(\mathbf{1})$ & $\begin{array}{c}\mathrm{R}=\text { neopentyl } \\
\text { (2) }\end{array}$ & $\mathrm{R}=n \mathrm{Bu}(\mathbf{3})$ & $\begin{array}{l}\text { LADH- } \\
\text { CNAD }^{\mathrm{a}}\end{array}$ \\
\hline $\mathrm{Zn}(1)-\mathrm{N}(1)(\AA)$ & $2.091(6)$ & $2.101(5)$ & $2.145(2)$ & 2.0 \\
\hline $\mathrm{Zn}(1)-\mathrm{Cl}(1)(\AA)$ & $2.210(2)$ & $2.2301(15)$ & $2.1930(9)$ & $2.2^{\mathrm{a}}$ \\
\hline $\mathrm{Zn}(1)-\mathrm{S}(1)(\AA)$ & $2.303(2)$ & $2.2937(16)$ & $2.2822(9)$ & 2.3 \\
\hline $\mathrm{Zn}(1)-\mathrm{S}(2)(\AA)$ & $2.307(2)$ & $2.3146(16)$ & $2.3431(9)$ & 2.3 \\
\hline $\mathrm{S}(1)-\mathrm{C}(6)(\AA)$ & $1.710(8)$ & $1.706(5)$ & $1.710(3)$ & \\
\hline $\mathrm{N}(1)-\mathrm{Zn}(1)-\mathrm{Cl}(1)$ & $111.96(17)$ & $111.80(13)$ & $113.92(7)$ & 136 \\
\hline $\mathrm{N}(1)-\mathrm{Zn}(1)-\mathrm{S}(2)$ & $96.66(17)$ & $97.83(13)$ & $94.07(7)$ & 106 \\
\hline $\mathrm{Cl}(1)-\mathrm{Zn}(1)-\mathrm{S}(2)$ & $117.01(8)$ & $118.76(13)$ & $108.92(4)$ & $97^{\mathrm{a}}$ \\
\hline $\mathrm{N}(1)-\mathrm{Zn}(1)-\mathrm{S}(1)$ & $95.53(17)$ & $94.98(13)$ & $96.01(7)$ & 97 \\
\hline $\mathrm{Cl}(1)-\mathrm{Zn}(1)-\mathrm{S}(1)$ & $120.44(9)$ & $117.41(6)$ & $123.85(4)$ & $98^{\mathrm{a}}$ \\
\hline $\mathrm{S}(2)-\mathrm{Zn}(1)-\mathrm{S}(1)$ & $110.36(8)$ & $111.46(6)$ & $115.26(3)$ & 125 \\
\hline
\end{tabular}

\footnotetext{
${ }^{\mathrm{a}}$ Instead of $\mathrm{Cl}$ atom in $\mathrm{LADH}-\mathrm{CNAD}$, this entry refers to $\mathrm{N} 5_{\mathrm{N}}$ atom of CNAD pyridine ring.

\subsection{Cyclic Voltammetry of Ligand Precursors and Comparison of Cyclic Voltammetry}

\section{Results to Gaussian Calculations.}

The bis-thione ligand precursor, $\mathbf{2} \mathbf{b}$, was studied by cyclic voltammetry, as part of its characterization. This compound shows a reversible oxidation wave at $1289 \mathrm{mV}$ and a reduction wave at $-2367 \mathrm{mV}$ by cyclic voltammetry in DMSO (Figure 2 ). The oxidation and reduction occurred at the same potential when two consecutive cycles were collected. 
Thus, the ligand precursor itself is stable with respect to repeated oxidations and reductions. We performed Gaussian calculations to further understand our cyclic voltammetry results.

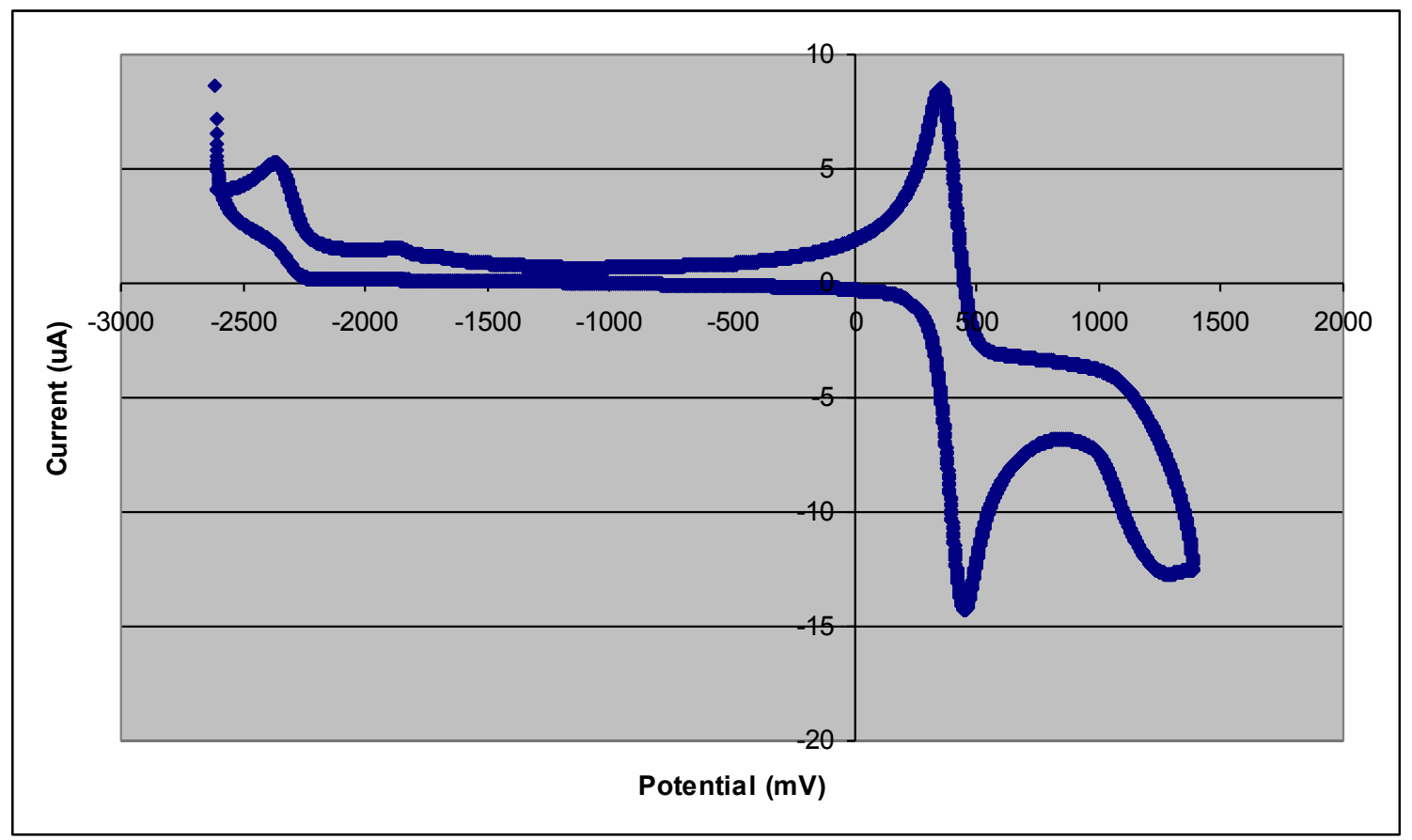

Figure 2. Cyclic Voltammogram of $\mathbf{2 b}$ in DMSO $(2 \mathrm{mM})$ with $0.2 \mathrm{M}$ TBAF. The scan rate was $100 \mathrm{mV} / \mathrm{s}$ with ferrocene $\left(\mathrm{E}_{1 / 2}=400 \mathrm{mV}\right)$ used as an internal standard.

Our Gaussian calculations agree quite well with the experimentally observed oxidation potential for the pincer ligand precursor. Under the various point groups considered, the computed $\Delta \mathrm{G}$ values are $1297 \mathrm{mV}\left(\mathrm{C}_{\mathrm{s}}\right), 1304 \mathrm{mV}\left(\mathrm{C}_{2}\right)$, and $1420 \mathrm{mV}$ $\left(\mathrm{C}_{2 \mathrm{v}}\right)$. While all three of these potentials are close to that found experimentally (1289 $\mathrm{mV})$, the $\Delta \mathrm{G}$ value found when the ligand is modeled under $\mathrm{C}_{\mathrm{s}}$ symmetry is the closest. As can be seen from the contour plot of the HOMO shown in Figure 3, the electron is removed from a $\pi$-type orbital that is largely based on the thioimidazolyl portions of the molecule. Upon oxidation, the main structural change observed in the optimized structures is an increase in the dihedral angle between the thioimidazolyl and pyridinyl 
sections of the molecule. Under $\mathrm{C}_{\mathrm{s}}$ symmetry, the dihedral angle is determined to be $21.3^{\circ}$ for the neutral system and $38.5^{\circ}$ for the cation, which indicates that the sulfur atoms are closer to each other in the oxidized compound than in the neutral system. Given that the sulfurs of the thioimidazole rings are closest to each other under $\mathrm{C}_{\mathrm{s}}$ symmetry than under the $\mathrm{C}_{2}$ or $\mathrm{C}_{2 \mathrm{v}}$ point groups and that similar systems are known to form disulfides upon oxidation [33], the excellent agreement between the experimental oxidation potential $(1289 \mathrm{mV})$ and the value calculated by Gaussian $(1297 \mathrm{mV})$ indicates an acceptable level of modeling for this system.

As for the one-electron reduction of the pincer ligand, the computed $\Delta \mathrm{G}$ values $\left(-5734 \mathrm{mV}\left(\mathrm{C}_{\mathrm{s}}\right),-5720 \mathrm{mV}\left(\mathrm{C}_{2}\right)\right.$, and $\left.-5748 \mathrm{mV}\left(\mathrm{C}_{2 \mathrm{v}}\right)\right)$ do not match the potential observed experimentally $(-2367 \mathrm{mV})$, suggesting that the reduction wave does not correspond with a simple one-electron reduction of the ligand precursor. The contour plot of the LUMO, shown in Figure 3, indicates that the electron is added into a $\pi$-type orbital that is distributed across all portions of the molecule. Upon reduction, the ligand is flat with a calculated thioimidazolyl-pyridinyl dihedral angle of $0.0^{\circ}$, which can be understood through consideration of the $\mathrm{N}-\mathrm{C} \pi$ bonding nature between the thioimidazolyl and pyridinyl fragments of the molecule. 

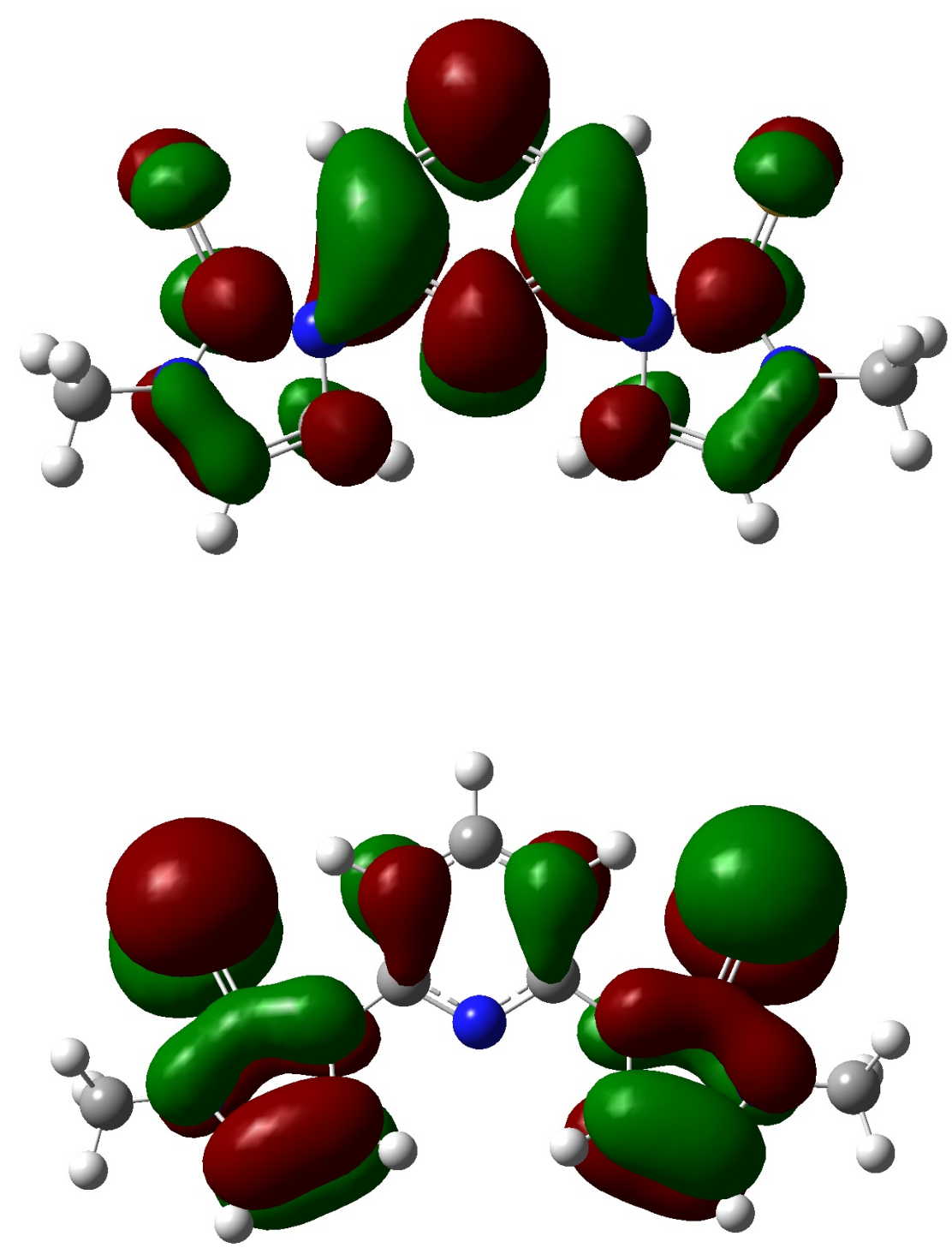

Figure 3. Orbital contour plots for the LUMO (top) and HOMO (bottom) of the methyl-substituted analogue of $\mathbf{2} \mathbf{b}$.

\subsection{Density Functional Theory Calculations to Understand Geometry About the Zinc}

\section{Center}

Because tridentate pincer ligand precursors are known to coordinate to metals in either a meridional or facial fashion, we were interested in understanding the energy 
difference between a pseudo-tetrahedral complex (facial coordination of pincer ligand) and a pseudo-square planar complex (meridional coordination of pincer ligand) of this zinc pincer system. Pincer ligands have been reported to coordinate in a meridional fashion to $\mathrm{d}^{8} \mathrm{Pd}(\mathrm{II})$ and $\mathrm{Pt}(\mathrm{II})$ centers to yield systems that possess a pseudo-square planar environment at the metal center [17]. Given the ability of the ligand precursor systems $\mathbf{1 b}, \mathbf{2} \mathbf{b}$, and $\mathbf{3 b}$ to establish $\pi$-conjugation across multiple rings when flat, we were interested in understanding whether such electronic influences would enforce a square planar environment upon the metal center or if the preference of $\mathrm{d}^{10} \mathrm{Zn}(\mathrm{II})$ to attain a tetrahedral coordination sphere would predominate.

Our calculations show that a pseudo-tetrahedral geometry is preferred for a $\mathrm{d}^{10}$ zinc system with a ligand precursor set that is analogous to that found in complexes 1-3 (Figure 1 and Table 1). The system with the $\mathrm{Zn}$ center in a pseudo-square planar environment and $\mathrm{C}_{2}$ molecular geometry overall is calculated to be $18.3 \mathrm{kcal} / \mathrm{mol}$ less stable than the pseudo-tetrahedral structure as depicted in Figure 4. The pseudo-square planar system with $\mathrm{C}_{2 \mathrm{v}}$ symmetry is $43.8 \mathrm{kcal} / \mathrm{mol}$ less stable than the pseudo-tetrahedral structure. Analysis of the $\mathrm{C}_{2}$ system finds that it has one vibration with an imaginary frequency (28.03i), the $\mathrm{C}_{2 \mathrm{v}}$ system possesses four (44.16i, 62.88i, 123.07i, and 138.96i $\mathrm{cm}^{-1}$ ), and the $\mathrm{C}_{\mathrm{s}}$ system has none in agreement with the observation of pseudotetrahedral systems for compounds 1-3. Visualization with GaussView of several of the imaginary frequencies for the $\mathrm{C}_{2 \mathrm{v}}$ system shows that they represent torsion of the ligand set away from being planar and toward the geometry adopted in the pseudo-tetrahedral structure. 
Zn-Cl: $2.17 \AA$

Zn-S: $2.22 \AA$

$\mathrm{Zn}-\mathrm{N}: 3.32 \AA$



Zn Geometry / Molecular Point Group

Figure 4. Optimized pseudo-tetrahedral and pseudo-square planar structures from DFT calculations. Select optimized bond distances are provided.

Various bond lengths and angles found in the three optimized structures are worth note. The $\mathrm{Zn}-\mathrm{S}, \mathrm{Zn}-\mathrm{Cl}$, and $\mathrm{Zn}-\mathrm{N}$ bond lengths of the pseudo-tetrahedral structure are in good agreement with those given for 1-3 in Table 1. Constraining the pincer precursor ligand to be planar to enforce a pseudo-square planar environment around the $\mathrm{Zn}$ center under $\mathrm{C}_{2 \mathrm{v}}$ symmetry requires the $\mathrm{Zn}-\mathrm{N}$ bond length to elongate to $3.32 \AA$, which is nearly $1.2 \AA$ longer than was calculated for the pseudo-tetrahedral structure. Furthermore, the bond angles at the $\mathrm{Zn}$ center $\left(\mathrm{N}-\mathrm{Zn}-\mathrm{S} 76.9^{\circ}\right)$ show that the donor atoms are reasonably 
well aligned with the filled $\mathrm{Zn} \mathrm{d}_{\mathrm{x} 2-\mathrm{y} 2}$ orbital, thereby providing instability to this structure. The pseudo-square planar system with $\mathrm{C}_{2}$ symmetry has a $\mathrm{Zn}-\mathrm{N}$ bond of a similar length (3.27 $\AA$ ), but given that the ligand can flex more freely than it can under $\mathrm{C}_{2 \mathrm{v}}$ symmetry, the optimized $\mathrm{N}-\mathrm{Zn}-\mathrm{S}$ bond angle of $65.1^{\circ}$ allows relaxation of the $\sigma$-donor atoms away from the $\mathrm{Zn} \mathrm{d}_{\mathrm{x} 2-\mathrm{y} 2}$ orbital and therefore a more stable structure than found for the $\mathrm{C}_{2 \mathrm{v}}$ system.

We next chose to calculate the energy differences between the pseudo-tetrahedral and pseudo-square planar systems with the ligand set split into three monodentate fragments. Doing so allows us to separate the influence of the $\mathrm{d}^{10}$ electronic population of the metal center from the coordination environment imposed by a unified tridentate SNS ligand precursor set. Shown in Figure 5 are the optimized pseudo-tetrahedral and pseudo-square planar structures in which the pyridine and imidazole fragments have been allowed to attain the most stable orientations possible within the constraints of the imposed molecular symmetries. In these structures, the pyridine rings are not connected to the imidazoles. No imaginary frequencies were found for the pseudo-tetrahedral $\mathrm{C}_{\mathrm{s}}$ structure while five $\left(18.32 \mathrm{i}, 21.91 \mathrm{i}, 24.28 \mathrm{i}, 28.34 \mathrm{i}, 45.73 \mathrm{i} \mathrm{cm}^{-1}\right)$ were found for the $\mathrm{C}_{2 \mathrm{v}}$ pseudo-square planar system and four $\left(7.77 \mathrm{i}, 9.81 \mathrm{i}, 19.52 \mathrm{i}, 52.57 \mathrm{i} \mathrm{cm}{ }^{-1}\right)$ were located for the $\mathrm{C}_{2}$ pseudo-square planar system. 


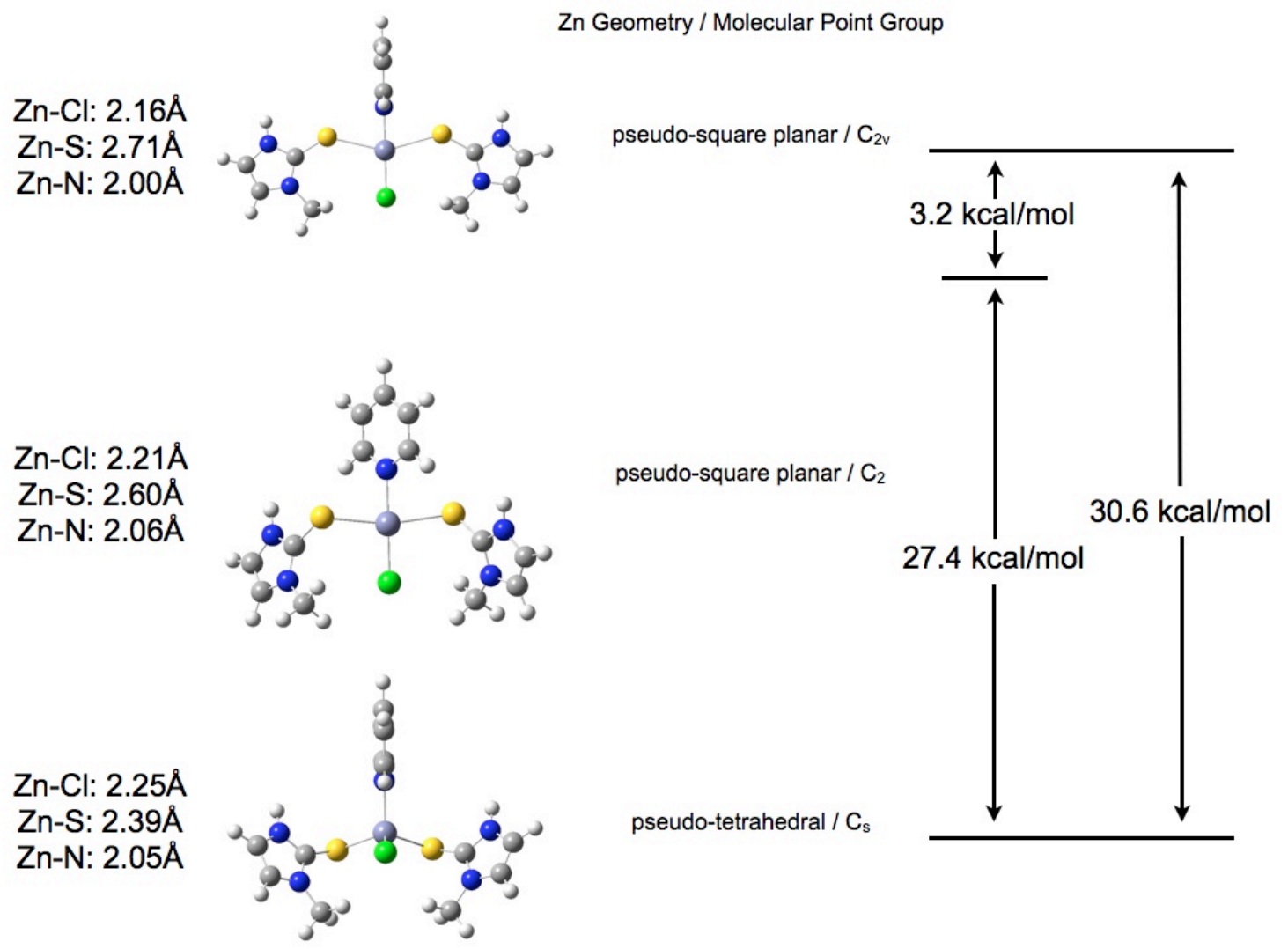

Figure 5. Optimized pseudo-tetrahedral and pseudo-square planar structures from DFT calculations with the pyridine ring and imidazole moities in optimal configurations. Select optimized bond distances are provided.

Comparison of the systems that contain monodentate ligands with those that have a tridentate ligand reveals interesting structural information. The calculated $\mathrm{Zn}-\mathrm{Cl}, \mathrm{Zn}-\mathrm{S}$, and $\mathrm{Zn}-\mathrm{N}$ bond lengths for the pseudo-tetrahedral systems are quite similar under both ligand environments. However, the $\mathrm{C}_{2 \mathrm{v}}$ pseudo-square planar system has comparatively short $\mathrm{Zn}-\mathrm{N}$ and long $\mathrm{Zn}-\mathrm{S}$ bonds in the tridentate ligand environment while the structural constraints of the single tridentate ligand impose a long $\mathrm{Zn}-\mathrm{N}$ bond and relatively short 
$\mathrm{Zn}-\mathrm{S}$ bonds on the molecule. The geometric requirements of a single, flat SNS ligand precursor therefore impose an additional $13.2 \mathrm{kcal} / \mathrm{mol}$ penalty $(43.8 \mathrm{kcal} / \mathrm{mol}-30.6$ $\mathrm{kcal} / \mathrm{mol}$ ) upon the $\mathrm{C}_{2 \mathrm{v}}$ pseudo-square planar structure relative to the $\mathrm{C}_{\mathrm{s}}$ pseudotetrahedral system.

On the whole, the relative stabilities of the pseudo-tetrahedral and pseudo-square planar systems are the same for this coordination environment about zinc whether the ligand set is a single tridentate SNS system or is broken into three separate units. The preference of a $\mathrm{d}^{10} \mathrm{Zn}$ center to attain a tetrahedral local environment trumps any stabilization gained by removal of constraints within the ligand set.

\subsection{Reactivity}

Having established a synthetic protocol for these metalloenzyme models and gained an understanding of their structural characteristics, we turned our attention to probing their stoichometric activity. We chose 4-nitrobenzaldehyde, an electron-poor aldehyde, to screen the ability of 1-3 to reduce such a system in the presence of the hydrogen donor BNAH (eq. 1). BNAH was prepared following a known literature procedure and is the reagent of choice to model NADH [25]. ${ }^{1} \mathrm{H}$ NMR spectroscopy was used to follow the disappearance of the aldehyde proton $(\delta 10.2 \mathrm{ppm})$ and the shifting of the aromatic $\mathrm{C}-\mathrm{H}$ protons in the alcohol product $(\delta 8.24 \mathrm{ppm})$. The aromatic proton resonance of the

alcohol product did not overlap with the ${ }^{1} \mathrm{H}$ NMR resonances of either the starting materials or the products or the zinc complex. In all of the reactivity experiments, 0.1 mmol of aldehyde, $0.1 \mathrm{mmol}$ zinc precursor or $0.2 \mathrm{mmol}$ of $\mathrm{ZnCl}_{2}$, and $0.2 \mathrm{mmol} \mathrm{BNAH}$ were used. When $\mathrm{ZnCl}_{2}$ was added, the $\mathrm{ZnCl}_{2}$ was the only zinc compound in the reaction mixture. In the control reactions, two equivalents of $\mathrm{ZnCl}_{2}$ were used because in 
complexes 1-3, there are two zinc ions (one in the cation and one in the anion) ion per neutral compound. The products were detected by ${ }^{1} \mathrm{H}$ NMR by comparison with authentic material. In no case was there any indication of reduction of nitro substituents. Table 3 illustrates the reactivity data for $\mathbf{1 - 3}$ as well as for $\mathrm{ZnCl}_{2}$ and the ligand precursor.<smiles>O=Cc1ccc([N+](=O)[O-])cc1</smiles>
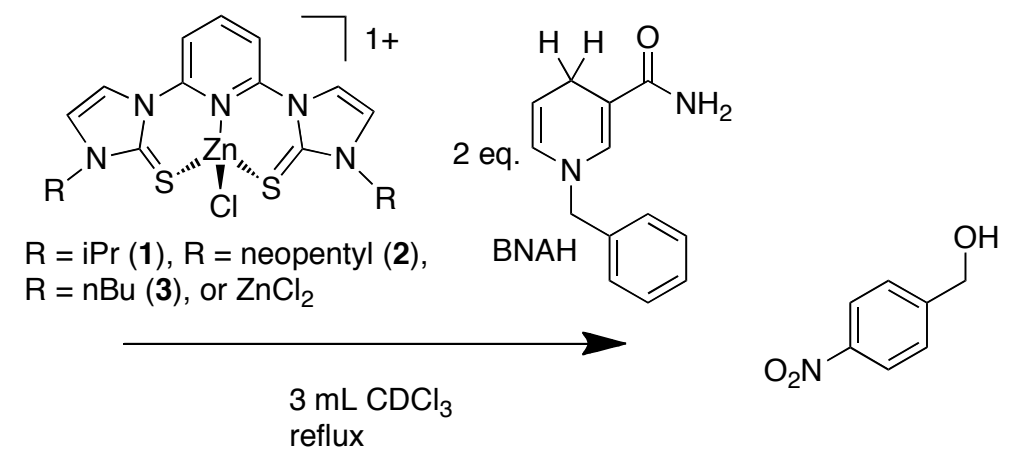

(eq. 1)

Table 3. Reactivity Data for 1-3

\begin{tabular}{cccc}
\hline Entry & Zn Complex & Time & Conversion (\%) \\
\hline 1 & None & $20 \mathrm{~h}$ & $<5$ \\
2 & $\mathbf{2 b}$ (bis thione ligand & $20 \mathrm{~h}$ & \\
3 & precursor) & & 15 \\
$\mathrm{ZnCl}_{2}(2$ eq. $)$ & $5 \mathrm{~h}$ & 13 \\
$\mathrm{ZnCl}_{2}{ }^{\mathrm{a}}(2$ eq. $)$ & $20 \mathrm{~h}$ & 18 \\
$\mathrm{ZnCl}_{2}(2$ eq. $)$ & $20 \mathrm{~h}$ & 42 \\
6 & $\mathbf{1}$ & $20 \mathrm{~h}$ & 48 \\
7 & $\mathbf{2}$ & $20 \mathrm{~h}$ & 33 \\
8 & $\mathbf{3}$ & $20 \mathrm{~h}$ & 42 \\
\hline
\end{tabular}


${ }^{a}$ Reaction was run under an $\mathrm{N}_{2}$ environment. The reaction was performed in an inert atmosphere glove box.

As shown in Table 3, zinc complexes 1-3 enhance the rate of the reaction for the reduction of 4-nitrobenzaldehyde when compared to $\mathrm{ZnCl}_{2}$ or ligand precursor. Based on a mechanism for LADH proposed by Berreau and co-workers, there is a hydrogen transfer between the co-factor and the substrate, which is coordinated to the zinc active site [32]. We also learned that $\mathrm{ZnCl}_{2}$ reacts stoichiometrically with electron poor aldehydes such as 4-nitrobenzaldhyde to yield alcohol product to a small extent (ca. $18 \%$ conversion). We suppose that $\mathrm{Zn}^{2+}$ acts as a Lewis acid catalyst in the reaction where $\mathrm{ZnCl}_{2}$ is utilized. Perhaps, the $\mathrm{Zn}^{2+}$ in the counteranion contributes to the reactivity that is shown for 1-3 in Table 3. Experiments are underway to prepare tridentate zinc SNS pincer complexes with a counter-anion that does not contain a zinc ion. We also wondered if an excess of $\mathrm{ZnCl}_{2}$ would enhance the rate of conversion of 4nitrobenzaldehyde. We saw $42 \%$ conversion of 4-nitrobenzaldehyde after $20 \mathrm{~h}$ when excess $\mathrm{ZnCl}_{2}$ (10 eq) was used. Thus, an excess of $\mathrm{ZnCl}_{2}$ and $\mathrm{BNAH}$ could be utilized to reduce 4-nitrobenzaldehyde.

Based on the data presented in Table 3, it appears that the choice of the R group is important as isopropyl and neopentyl groups gave a higher percent conversion than $n$ butyl. Larger R groups are needed to prepare zinc complexes that have increased solubility in organic solvents. The more sterically demanding $n$-butyl wingtip group, when compared to neopentyl or isopropyl, may shield the zinc metal center from interacting with BNAH and substrate. This is another example that illustrates that the 
identity of the wingtip group is a very important variable to consider when screening metal complexes for activity [23].

We also tried to reduce another electron poor aldehyde, pyridine-2-carboxaldehyde, in the presence of a stoichiometric amount of $\mathbf{1}$ or $\mathrm{ZnCl}_{2}$ (eq. 2).
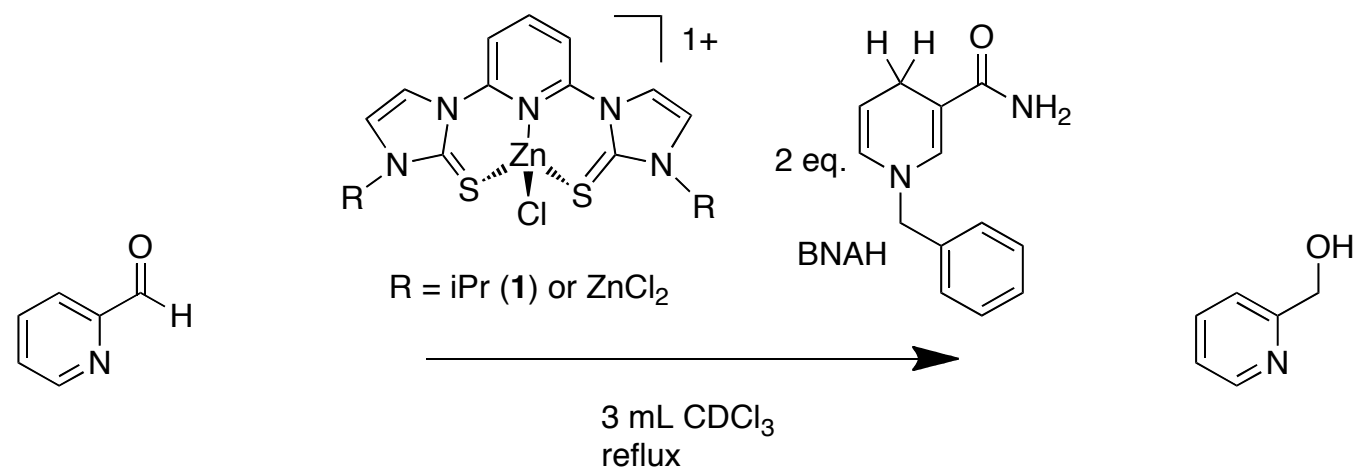

(eq. 2)

${ }^{1} \mathrm{H}$ NMR was used to follow the disappearance of the aldehyde proton ( $\left.\delta 10.2 \mathrm{ppm}\right)$ and the shifting of the aromatic $\mathrm{C}-\mathrm{H}$ protons in the alcohol product $(\delta 8.4 \mathrm{ppm})$. The aromatic proton resonance of the alcohol product did not overlap with the ${ }^{1} \mathrm{H}$ NMR resonances of either the starting materials or the products or the zinc complex. Table 4 illustrates the reactivity data for $\mathbf{1}$ as well as for $\mathrm{ZnCl}_{2}$.

Table 4. Reactivity Data for $\mathbf{1}$ for the reduction of pyridine-2-carboxaldehyde.

\begin{tabular}{cccc}
\hline Entry & Zn Complex & Time & Conversion (\%) \\
\hline 1 & None & $20 \mathrm{~h}$ & $<5$ \\
2 & $\mathbf{1}$ & $20 \mathrm{~h}$ & 35 \\
3 & $\mathrm{ZnCl}_{2}$ & $20 \mathrm{~h}$ & 19 \\
\hline
\end{tabular}

As shown in Table 4, zinc complex 1 enhances the rate of the reaction for the reduction of pyridine-2-carboxaldehyde when compared to $\mathrm{ZnCl}_{2}$. 
As seen in Tables 3 and 4, enhancement for the reduction of 4-nitrobenzaldehyde or pyridine-2-carboxaldehyde was observed for complexes 1-3. Stoichiometric conversion to the alcohol product was not observed for either 4-nitrobenzaldehyde or pyridine-2carboxaldehyde. Based on the mechanism proposed by Berreau and co-workers [32], the low activity of complexes 1-3 could be due to the slow hydrogen transfer between the cofactor and the substrate, which is coordinated to the zinc active site. More importantly, the alcohol product may inhibit the reaction. As the alcohol is formed, it may coordinate to the zinc metal center as the reaction progresses, and thereby hinder the reaction.

\section{Conclusions}

A series of $\mathrm{Zn}(\mathrm{II})$ compounds containing the SNS facial coordination of a tridentate pincer ligand was prepared and characterized. The tridentate SNS pincer ligand precursors and zinc complexes used in this work have provided new insights in the field of bioinorganic modeling chemistry. The zinc complexes serve as models for the zinc active site in liver alcohol dehydrogenase. The SNS zinc pincer complexes adopt pseudo-tetrahedral geometry and have a SNS coordination environment about the zinc center like LADH and react with BNAH to reduce electron poor aldehydes. The reactivity of $\mathbf{1 - 3}$ is not optimal when compared to LADH. It remains a challenge to synthesize a neutral zinc complex with a tridentate ligand with SNS donor atoms that

yield reactivity that is comparable to $\mathrm{LADH}$.

Gaussian calculations were performed to prove various structural and electronic properties of these compounds. The computed oxidation potentials matches well with what is observed experimentally while the calculated reduction potential indicates that 
the experimental reduction wave does not correspond to a simple one-electron reduction of the ligand precursor without other reactivity occurring. DFT calculations were also performed to better understand why the geometry about the zinc center is pseudotetrahedral rather than pseudo-square planar, which is seen for most pincer complexes. Overall, the relative stabilities of the pseudo-tetrahedral and pseudo-square planar systems are the same for this coordination environment whether the ligand set is a single tridentate SNS system or is broken into three separate units. The preference of a $\mathrm{d}^{10} \mathrm{Zn}$ center to attain a tetrahedral local environment trumps any stabilization gained by removal of constraints within the ligand set.

\section{Supporting Information:}

The ${ }^{1} \mathrm{H},{ }^{13} \mathrm{C}$ and HSQC NMR spectra of $\mathbf{2 a}, \mathbf{2} \mathbf{b}, \mathbf{3} \mathbf{b}$, and $\mathbf{1 - 3}$ and mass spectra of $\mathbf{1 - 3}$ are provided. IR spectra for $\mathbf{2 b}$ and $\mathbf{3 b}$ and crystallographic details of $\mathbf{1 - 3}$ are also given.

\section{Acknowledgement:}

This work was supported by generous funding from The Fairfield University Science Institute (JRM), Fairfield University Start-up Funding (JRM), a Fairfield University Research Grant (JRM), and a NTID Faculty Evaluation and Development Grant (MAL). JRM would like to thank Prof. Matthew A. Kubasik, Prof. L. Kraig Steffen, and Prof. Swapan Jain, for helpful suggestions.

\section{References:}

[1] R.H. Holm, P. Kennepohl, E.I. Solomon, Chem. Rev., 1996, 96, 2239-2314.

[2] J.A. Ibers, R.H. Holm, Science, 1980, 209, 223-235. 
[3] (a) W.N. Lipscomb, N. Sträter, Chem. Rev. 1996, 96, 2375-2433. (b) E. Kimura, T. Koike, M. Shionoya, Structure and Bonding, 1997, 89, 1-28.

[4] K.K. Kannan, B. Nostrand, K. Fridborg, S. Lovgren, A. Ohlsson, M. Petef, Proc. Natl. Acad. Sci, USA, 1975, 72, 51.

[5] A. Meibner, W. Haehnel, H. Vahrenkamp, Chem. Eur. J, 1997, 3, 261-267.

[6] A. Dolega, Coord. Chem. Rev., 2010, 254, 916-937.

[7] A. Dolega, A. Pladzyk, K. Baranowska, J. Jezierska, Inorg. Chim. Acta, 2009, 362, 5085-5096.

[8] L. Cronin, P.H. Walton, Chem Commun, 2003, 1572-1573.

[9] L.M. Berreau, M.M. Makowska-Grzyska, A.M. Arif, Inorg Chem, 2001, 40, $2212-$ 2213.

[10] R.M.Kellogg, R.P. Hof, J Chem Soc, Perkin Trans 1, 1996, 1651-1657.

[11] S.C. Shoner, K.J. Humphreys, D. Barnhart, J.A. Kovacs, Inorg. Chem, 1995, 34, 5933-5934.

[12] (a) B. Kaptein, G. Barf, R.M. Kellogg, F. Van Bolhuis, J. Org. Chem, 1990, 55, 1890-1891. (b) B. Kaptein, L.Wang-Griffen, G. Bart, R.M. Kellogg, J Chem. Soc Chem Commun, 1987, 1457-1459.

[13] D.T. Corwin, R. Fikar, S.A. Koch, Inorg. Chem, 1987, 26, 3079-3080.

[14] (a) C. Kimblin, T. Hascall, G. Parkin, Inorg. Chem., 1997, 36, 5680-568. (b) C. Kimblin, B.M. Bridgewater, D.G. Churchill, G. Parkin, Chem. Commun; 1999, 23012302.

[15] (a) R. Walz, H. Vahrenkamp, Inorg. Chim. Acta, 2001, 314, 58-62. (b) Y.H. Zhang, H. Vahrenkamp, Inorg. Chim. Acta, 2003, 351, 201-206.

[16] (a) M. Albrecht, G. van Koten, Angew. Chem. Int. Ed., 2001, 40, 3750-3781. (b) A.T. Normand, K.J. Cavell, Eur. J. Inorg. Chem., 2008, 2781-2800.

[17] D. Morales-Morales, C.M. Jensen, eds. The Chemistry of Pincer Compounds, Elsevier: New York, 2007.

[18] (a) C. Kimblin, T. Hascall, G. Parkin, Inorg. Chem., 1997, 36, 5680-568. (b) C. Kimblin, B.M. Bridgewater, D.G. Churchill, G. Parkin, Chem. Commu, 1999, 23012302. (c) C. Bergquist, G. Parkin, Inorg. Chem., 1999, 38, 422-423. (d) G. Parkin, Chem. Rev, 2004, 104, 699-767. (e) B.M. Bridgewater, T. Fillebeen, R.A. Friesner, G. Parkin, J. Chem. Soc., Dalton Trans, 2000, 4494-4496. (f) J.G. Melnick, A. Docrat, G. 
Parkin, Chem. Commun, 2004, 2870-2871. (g) C. Kimblin, B.M. Bridgewater, D.G. Churchill, T. Hascall, G. Parkin, Inorg. Chem, 2000, 39, 4240-4243.

[19] (a) M. Tessmer, M. Shu, H. Vahrenkamp, Inorg. Chem., 2001, 40, 4022-4029. (b) J. Seebacher, M. Shu, H. Vahrenkamp, Chem. Commun; 2001, 1026-1027. (c) Y.H. Zhang, H. Vahrenkamp, Inorg. Chim. Acta, 2003, 351, 201-206. (d) M.M. Ibrahim, M. Shu, H. Vahrenkamp, Eur. J. Inorg. Chem, 2005, 1388-1397. (e) R. Walz, H. Vahrenkamp, H. Inorg. Chim. Acta, 2001, 314, 58-62. (f) M. Rombach, J. Seebacher, M. Ji, G. Zhang, G. He, M. Ibrahim, B. Benkmil, H. Vahrenkamp, Inorg. Chem, 2006, 45, 4571-4575. (g) M. Ibrahim, J. Seebacher, G. Steinfeld, H. Vahrenkamp, Inorg. Chem, 2005, 44, 8531-8538.

[20] Gridnev, A.A.; Mihaltseva, I.M. Synth. Commun., 1994, 24, 1547.

[21] J.A. Loch, M.A. Albrecht, E. Peris, J. Mata, J.W. Faller, R.H. Crabtree, Organomet., 2002, 21(4), 700-706.

[22] E. Kuwano, M. Kikuchi, M. Eto, Agricultural and Biological Chem, 1988, 52(6), 1619-1620.

[23] M. Albrecht, J.R. Miecznikowski, A. Samuel, J.W. Faller, R.H. Crabtree, Organomet, 2002, 21(17), 3596-3604.

[24] W.-G. Jia, Y.-B. Huang, G.-X. Jin, G-X. J. Organomet. Chem, 2009, 694 (25), 4008-4013.

[25] J. Lutz, F. Hollmann, T.V. Ho, A. Schnyder, R. Fish, A. Schmid, J. Organomet. Chem, 2004, 689, 4783-4790.

[26] M.J. Frisch, G.W. Trucks, H.B. Schlegel, G.E. Scuseria, M.A. Robb, J.R. Cheeseman, J. A. Montgomery Jr., T. Vreven, K.N. Kudin, J.C. Burant, J. M. Millam, S. S. Iyengar, J. Tomasi, V. Barone, B. Mennucci, M. Cossi, G. Scalmani, N. Rega, G.A. Petersson, H. Nakatsuji, M. Hada, M. Ehara, K. Toyota, R. Fukuda, J. Hasegawa, M. Ishida, T. Nakajima, Y. Honda, O. Kitao, H. Nakai, M. Klene, X. Li, J.E. Knox, H.P. Hratchian, J.B. Cross, V. Bakken, C. Adamo, J. Jaramillo, R. Gomperts, R.E. Stratmann, O. Yazyev, A.J. Austin, R. Cammi, C. Pomelli, J.W. Ochterski, P.Y. Ayala, K. Morokuma, G.A. Voth, P. Salvador, J.J. Dannenberg, V.G. Zakrzewski, S. Dapprich, A.D. Daniels, M.C. Strain, O. Farkas, D.K. Malick, A.D. Rabuck, K. Raghavachari, J.B. Foresman, J. V. Ortiz, Q. Cui, A.G. Baboul, S. Clifford, J. Cioslowski, B. B. Stefanov, G. Liu, A. Liashenko, P. Piskorz, I. Komaromi, R.L. Martin, D.J. Fox, T. Keith, M.A. AlLaham, C.Y. Peng, A. Nanayakkara, M. Challacombe, P.M.W. Gill, B. Johnson, W. Chen, M.W. Wong, C. Gonzalez, J.A. Pople, Gaussian 03, Revision E.01; Gaussian, Inc.: Wallingford, CT, 2004.

[27] (a) W.R. Fawcett, Langmuir, 2008, 24, 9868. (b) L.E. Roy, E. Jakubikova, G. Guthrie, E.R. Batista, J. Phys. Chem. A, 2009, 113, 6745-6750. 
[28] E. Peris, J.A. Loch, J. Mata, R.H. Crabtree, Chem Commun, 2001, 201-202.

[29] A. Caballero, E. Díez-Barra, F.A. Jalón, S. Merino, J. Tejeda, J. Organomet. Chem., 2001, 617-618, 395-398.

[30] B.V. Trzhtsinskaya, N.D. Abramova, J Sulfur Chemistry, 1991, 10(4), 389-421.

[31] H. Li, W.H. Hallows, J.S. Punzi, K.W. Pankiewicz, K.A. Watanabe, B.M. Goldstein, Biochemistry, 1994, 33, 11734-11744.

[32] M.M. Makowska-Grzyska, P.C. Jeppson, R.A. Allred, A.M. Arif, L.M. Berreau. Inorg. Chem, 2002, 41, 4872-4887.

[33] A. Suszka, Polish J of Chemistry, 1980, 54, 2289-2295. 